\title{
DIFFERENTIAL GERSTENHABER ALGEBRAS OF GENERALIZED COMPLEX STRUCTURES*
}

\author{
DANIELE GRANDINI ${ }^{\dagger}$, YAT-SUN POON ${ }^{\ddagger}$, AND BRIAN ROLLE ${ }^{\S}$
}

\begin{abstract}
Associated to every generalized complex structure is a differential Gerstenhaber algebra (DGA). When the generalized complex structure deforms, so does the associated DGA. In this paper, we identify the infinitesimal conditions when the DGA is invariant as the generalized complex structure deforms. We prove that the infinitesimal condition is always integrable. When the underlying manifold is a holomorphic Poisson nilmanifolds, or simply a group in the general, and the geometry is invariant, we find a general construction to solve the infinitesimal conditions under some geometric conditions. Examples and counterexamples of existence of solutions to the infinitesimal conditions are given.
\end{abstract}

Key words. DGA, generalized complex, holomorphic Poisson, nilmanifolds.

AMS subject classifications. Primary: 53D18; Secondary: 16E45, 22E25, 32G05, 53D17.

1. Introduction. A few years ago, the second author computed the weak Frobenius structure on the moduli space of the Barannikov-Kontsevich's extended deformation [2] of the complex structure on a primary Kodaira surface [26]. Among other observations, one could see from [26, Table (45)] that the restriction of the weak Frobenius structure to the even part of the extended moduli space is trivial. The parameter space of the even part of the extended moduli at the unperturbed point is contained in

$$
\oplus_{k=\text { even }} H_{J}^{k}, \quad \text { where } \quad H_{J}^{k}=\oplus_{p+q=k} H^{q}\left(M, \wedge^{p} T^{1,0}\right)
$$

and $T^{1,0}$ is the holomorphic tangent bundle of the complex manifold $M$. The computation in [26] dwells in the fact that the primary Kodaira surface was chosen to be a nilmanifold and the complex structure he worked with is invariant. Along the line of thoughts in [25] [16], the Dolbeault cohomology could be computed by means of algebraic methods.

Thanks to the work of Hitchin [18] and Gualtieri [17], it is now well known that the degree-2 portion of the extended deformation is realized by deformation of generalized geometry. While we will provide further details on generalized geometry in Section 2, at this stage we simply note that the parameter space of generalized deformation is the degree- 2 portion of extended deformation.

$$
H_{J}^{2}=H^{0}\left(M, \wedge^{2} T^{1,0}\right) \oplus H^{1}\left(M, T^{1,0}\right) \oplus H^{2}(M, \mathcal{O})
$$

where $\mathcal{O}$ is the structure sheaf of the complex manifold $M$.

The key ingredient in constructing the weak Frobenius structure on extended deformation is a variation of the exterior product structure when the concerned cohomology spaces vary. However, it is also known that the differential geometric object controlling the extended deformations is the differential Gerstenhaber algebras (DGA)

\footnotetext{
*Received March 5, 2012; accepted for publication November 9, 2012.

${ }^{\dagger}$ Department of Mathematics, University of California at Riverside, Riverside CA 92521, U.S.A.

${ }^{\ddagger}$ Department of Mathematics, University of California at Riverside, Riverside CA 92521, U.S.A. (ypoon@ucr.edu). Partially supported by NSF DMS-0906264 and Mathematical Sciences Center of Tsinghua University.

$\S$ Department of Mathematics, University of California at Riverside, Riverside CA 92521, U.S.A.
} 
associated to each (extended) complex structure [5] [23] [24]. We will provide necessary details on the construction of DGAs in Section 3. This structure contains the exterior differential algebra as a sub-structure. In this context, we could paraphrase a result of [26] in a context of generalized complex geometry, and say that the exterior differential algebras along a generalized deformation of a primary Kodaira surface is rigid, meaning that all the exterior differential algebras are quasi-isomorphic to the unperturbed one. From this perspective, we seek a general understanding of the rigidity of the full differential Gerstenhaber algebra structures.

QUESTION 1. Suppose that $M$ is a manifold with generalized complex structure $J$. Let $\mathrm{DGA}(0)$ be the associated differential Gerstenhaber algebra. Suppose that $\Gamma(t)$ is a family of deformation of $J$ along generalized complex structure with parameter $t$, with associated differential Gerstenhaber algebra DGA $(t)$. Under what condition will $\operatorname{DGA}(t)$ be quasi-isomorphic to $\mathrm{DGA}(0)$ ?

The infinitesimal counter-part of $\Gamma(t)$ is $\Gamma_{1}$, which represents an element in the cohomology space $H_{J}^{2}$. If there is a quasi-isomorphism $\Phi(t)$, depending on $t$, we consider its infinitesimal version $\phi$. The pair $\Gamma_{1}$ and $\phi$ will be addressed as a compatible pair. Together, they have to satisfy a set of constraints as given in Definition 1 . The main result in Section 3 is Theorem 2, which states essentially that compatible pairs are always integrable. Therefore, answers to Question 1 above are reduced to infinitesimal level.

In identity (2), we see that there are three special kinds of deformations to analyze. Those from $H^{1}\left(M, T^{1,0}\right)$ are due to classical complex deformation theory. Those from $H^{2}(M, \mathcal{O})$ are due to $B$-field transformations if the underlying complex structure is Kählerian [17]. Therefore, we focus on those in the component $H^{0}\left(M, \wedge^{2} T^{1,0}\right)$. As we will explain later, this class of deformation is due to holomorphic Poisson structures, objects under investigation from various perspectives [13] [15] [19] [20]. If the holomorphic Poisson structure has full rank everywhere, it leads to a deformation from a classical complex structure $J$ to a symplectic structure $\Omega$. If the induced differential Gerstenhaber algebras along this deformation is rigid, then $\operatorname{DGA}(J)$ and $\operatorname{DGA}(\Omega)$ are quasi-isomorphic. It presents the complex manifold $(M, J)$ and the symplectic manifold $(M, \Omega)$ as a weak mirror pair in the sense of Merkulov [24]. An investigation on such possibility also motivates this paper. Therefore, in Section 4 we refine our analysis in Section 3 to holomorphic Poisson manifolds, and illustrate our theory with a computation on a Hopf surface.

For nilmanifolds, i.e. the compact quotient of simply connected nilpotent Lie groups, it is known for a very long time that the de Rham cohomology is given by invariant elements [25]. From our current perspective, the invariant DGA with an invariant symplectic structure on a nilmanifold is quasi-isomorphic to the full DGA of the symplectic structure. For a large class of nilmanifolds examples, we also know that the invariant DGA theory for invariant complex structures is quasiisomorphic to the DGA of the corresponding nilmanifolds [10] [16] [26] [28]. Therefore, we reduce the theory in the previous sections in terms of invariant objects on Lie algebras and develop a method to construct compatible pairs on a class of holomorphic Poisson algebras in Section 5. Finally, in Section 6 we analyze all non-trivial real fourdimensional examples. Among other observations, we conclude that the differential Gerstenhaber algebra structures are rigid when one deforms the complex structure on a Kodaira surface by a holomorphic Poisson structure. It extends the results in [26] on weak Frobenius structures, at least along the degree-2 direction of the extended moduli space. On the other hand, we also discover an example of holomorphic symplectic 
algebra on which there is no compatible pair. Therefore, a solution to Question 1 is non-trivial.

In this notes, we assume that readers are familiar with the concepts of Lie algebroids and Lie bialgebroids. Otherwise, [21] and [22] are our references. On Differential Gerstenhaber algebras, we rely on [22] and [26] for their formal aspects. For generalized complex structures, our references are [18] and [17]. Much of the computation in Section 5 and Section 6 could be found in the third author's thesis. Therefore, our presentation will be relatively sketchy.

2. Generalized complex structures. Let $M$ be a smooth connected manifold without boundary. Denote its tangent and cotangent bundle respectively by $T$ and $T^{*}$. If $V$ is a vector bundle on $M$, we denote its space of sections by $C^{\infty}(V)$. Generic vector fields will be denoted by $X$ and $Y$. One-forms are denoted by $\alpha$ and $\beta$. On the bundle $T \oplus T^{*}$, there is a natural pairing defined by

$$
\langle X+\alpha, Y+\beta\rangle=\frac{1}{2}(\alpha(Y)+\beta(X)) .
$$

As this pairing is non-degenerate, it identifies the bundle $T \oplus T^{*}$ to its dual. We choose the identification to be

$$
\sigma: T \oplus T^{*} \rightarrow\left(T \oplus T^{*}\right)^{*}, \quad \sigma(X+\alpha)(Y+\beta)=2\langle X+\alpha, Y+\beta\rangle .
$$

The Courant bracket [21] is the real bilinear map on $C^{\infty}\left(T \oplus T^{*}\right)$ defined by

$$
\llbracket X+\alpha, Y+\beta \rrbracket=[X, Y]+\mathcal{L}_{X} \beta-\mathcal{L}_{Y} \alpha-\frac{1}{2} d\left(\iota_{X} \beta-\iota_{Y} \alpha\right) .
$$

The Courant bracket, the non-degenerate pairing above, together with the natural projection on the tangent component make $T \oplus T^{*}$ a standard example of a Courant algebroid [11] [21].

An almost generalized complex structure is a real bundle map $J: T \oplus T^{*} \rightarrow T \oplus T^{*}$ such that $J \circ J=-$ identify and $J^{*}=-J$. Let $L$ be the bundle of $+i$-eigenvectors with respect to $J$ and over the complex numbers. With respect to the non-degenerate pairing, $L$ is maximal isotropic. So is its conjugate bundle $\bar{L}$. The choice of the tensorial object $J$ with the given prescription is equivalent to the choice of maximal isotropic subbundle $L$ such that $L \cap \bar{L}$ is trivial [17].

An almost generalized complex structure is said to be integrable if and only if the space $C^{\infty}(L)$ is closed under the Courant bracket. By complex conjugation, it is of course equivalent to $C^{\infty}(\bar{L})$ being closed. In such case, the structure $J$, or equivalently, either the bundle $L$ or the bundle $\bar{L}$ is said to be a generalized complex structure. It is now well known that complex structures in the classical sense are generalized complex. So are symplectic structures. For classical complex structure, the complexified tangent bundle splits into the direct sum of type $(1,0)$ and type $(0,1)$ vectors. Their related bundles are denoted by $T^{1,0}$ and $T^{0,1}$ respectively. Their dual bundles are denoted by $T^{*(1,0)}$ and $T^{*(0,1)}$. Then the corresponding bundles $L$ and $L^{*}$ are

$$
L=T^{1,0} \oplus T^{*(0,1)}, \quad L^{*} \cong \bar{L}=T^{0,1} \oplus T^{*(1,0)} .
$$

If $\omega$ is a symplectic form on the manifold $M$, then the pair

$$
L=\left\{X-i \iota_{X} \omega: X \in T\right\}, \quad L^{*} \cong \bar{L}=\left\{X+i \iota_{X} \omega: X \in T\right\}
$$


represents an example of a generalized complex structure.

Since $L$ is isotropic, the restriction of the Courant bracket on $L$ makes it a Lie algebroid whenever the generalized complex structure is integrable. As such, it has a Lie algebroid differential acting on the exterior algebra of the dual bundle [22].

$$
\partial: C^{\infty}\left(\wedge^{n} L^{*}\right) \rightarrow C^{\infty}\left(\wedge^{n+1} L^{*}\right) .
$$

Using the identification as given in (4), we identify $\bar{L}=L^{*}$. Then

$$
\partial: C^{\infty}\left(\wedge^{n} \bar{L}\right) \rightarrow C^{\infty}\left(\wedge^{n+1} \bar{L}\right) .
$$

Similarly, $\bar{L} \cong L^{*}$ is also a Lie algebroid. Its Lie algebroid differential is precisely the conjugation of the above operator:

$$
\bar{\partial}: C^{\infty}\left(\wedge^{n} L\right) \rightarrow C^{\infty}\left(\wedge^{n+1} L\right) .
$$

As noted in [21, Theorem 2.6], $(L, \bar{L})$ forms a Lie bialgebroid. It means that for any sections $\ell_{1}$ and $\ell_{2}$ of the bundle $L$,

$$
\bar{\partial} \llbracket \ell_{1}, \ell_{2} \rrbracket=\llbracket \bar{\partial} \ell_{1}, \ell_{2} \rrbracket-\llbracket \ell_{1}, \bar{\partial} \ell_{2} \rrbracket .
$$

Making use of [22, Theorem 7.5.2], we deduce that the space of sections of the exterior algebra generated by $L, C^{\infty}\left(\wedge^{\bullet} L\right)$ carries the structure of a differential Gerstenhaber algebra structure, with the Courant bracket, exterior product and Lie algebroid differential of $\bar{L} \cong L^{*}$. We denote it by

$$
D G A(J):=\left(C^{\infty}\left(\wedge^{\bullet} L\right), \llbracket-,-\rrbracket, \wedge, \bar{\partial}\right) .
$$

In this context, the bracket on $C^{\infty}\left(\wedge^{\bullet} L\right)$ is known as Schouten bracket [22].

The integrability implies that the restriction of the Courant bracket on $C^{\infty}\left(L^{*}\right)$ satisfies the Jacobi identity. In terms of the operator $\bar{\partial}$, it is equivalent to $\bar{\partial} \circ \bar{\partial}=0$. Therefore, $\bar{\partial}: C^{\infty}\left(\wedge^{n} L\right) \rightarrow C^{\infty}\left(\wedge^{n+1} L\right)$ determines a differential complex, and hence generates cohomology spaces. i.e. for all $k \geq 1$,

$$
H_{J}^{k}=\frac{\operatorname{ker} \bar{\partial}: \wedge^{k} L \rightarrow \wedge^{k+1} L}{\text { Image } \bar{\partial}: \wedge^{k-1} L \rightarrow \wedge^{k} L} .
$$

Given the identity (9), the cohomology spaces inherit a Gerstenhaber algebra structure.

When the generalized complex structure is a classical complex structure, one could verify that if $\bar{\omega}$ is a type $(0, k)$-form, then $\bar{\partial} \bar{\omega}$ is the classical $\bar{\partial}$-operator in complex analysis on $\mathbb{C}^{n}[28]$. On the other hand, if $Z$ is a $(1,0)$-vector field and $\bar{X}$ is a $(0,1)$-vector field, then

$$
\bar{\partial}_{\bar{X}} Z=[Z, \bar{X}]^{1,0} .
$$

This is precisely the Cauchy-Riemann operator [14] [27]. The cohomology of degree- $k$ in this case is

$$
H_{J}^{k}=\oplus_{p+q=k} H^{q}\left(M, \wedge^{p} T^{1,0}\right) .
$$

Using Dolbeault theory, the elements in these cohomology spaces are represented by $\bar{\partial}$-closed $(0, q)$-forms with coefficients in holomorphic $(p, 0)$-vector fields. 
On the other hand, if a generalized complex structure is defined by a symplectic form $\omega$, then

$$
\bar{\partial}\left(X-i \iota_{X} \theta\right)=-2 i d \iota_{X} \theta
$$

for all $X$ in $C^{\infty}\left(T_{\mathbb{C}}\right)$ [27]. In particular, the k-th cohomology of this complex is the k-th complexified de Rham cohomology of the manifold $M$.

As a subbundle of $\left(T \oplus T^{*}\right)_{\mathbb{C}}$, the bundle $L$ has a natural projection $\rho$ onto the direct summand $T_{\mathbb{C}}$. The type of a generalized complex structure at a point of the manifold $M$ is defined to be the complex co-dimension of the projection of $L$ in $T_{\mathbb{C}}$ over the concerned point [17]. From the description above, one sees that the type of a classical complex structure on a real $2 n$-dimensional manifold is equal to $n$. All symplectic structures are type-0 generalized complex structures.

3. Deformation of generalized complex structures. A deformation of a generalized complex structure is given by a section $\Gamma$ of $\wedge^{2} L$ [21] [17]. To be more precise,

$$
L_{\bar{\Gamma}}=\{\ell+\bar{\Gamma}(\ell): \ell \in L\}, \quad \text { and } \quad \bar{L}_{\Gamma}=\{\bar{\ell}+\Gamma(\bar{\ell}): \bar{\ell} \in \bar{L}\} .
$$

$L_{\bar{\Gamma}} \cap \bar{L}_{\Gamma}=\{0\}$ if and only if $\Gamma \circ \bar{\Gamma}$ does not have non-trivial fixed points [27]. The deformed generalized complex structure $\left(L_{\bar{\Gamma}}, \bar{L}_{\Gamma}\right)$ is integrable if and only if $\Gamma$ satisfies the Maurer-Cartan equation [21, Theorem 6.1]:

$$
\bar{\partial} \Gamma+\frac{1}{2} \llbracket \Gamma, \Gamma \rrbracket=0 .
$$

The infinitesimal version of the Maurer-Cartan equation is simply $\bar{\partial} \Gamma_{1}=0$. Therefore, it represents an element in the second cohomology $H_{J}^{2}$ of the differential Gerstenhaber algebra of the unperturbed generalized complex structure $J$.

3.1. Deformation of associated DGA. Let $\bar{\delta}$ be the Lie algebroid differential of $\bar{L}_{\Gamma}$. Due to our natural pairing (3), it acts on the conjugate bundle $L_{\bar{\Gamma}}$. Therefore, we have the new differential Gerstenhaber algebra

$$
D G A\left(J_{\Gamma}\right)=\left(\wedge^{\bullet} L_{\bar{\Gamma}}, \llbracket-,-\rrbracket, \wedge, \bar{\delta}\right) .
$$

Meanwhile, for $\Gamma$ sufficiently close to zero, $L$ and $\bar{L}_{\Gamma}$ are also transversal in $(T \oplus$ $\left.T^{*}\right)_{\mathbb{C}}$. By [21, Theorem 2.6], $L$ and $\bar{L}_{\Gamma}$ form a Lie bialgebroid. We could denote the Lie algebroid differential of the Lie algebroid $\bar{L}_{\Gamma}$ acting on $L$ by $\bar{\partial}_{\Gamma}$. Since $L_{\bar{\Gamma}}$ is simply the graph of the map $\bar{\Gamma}$, there is a natural map from $L$ to $L_{\Gamma}$. It enables one to identify the differential $\bar{\partial}_{\Gamma}$. The computation below is a consequence of [21, Theorem 2.6] and [21, Theorem 6.1]. It should be well known to experts. We outline a proof here for completeness. A complete proof for a case most relevant to this paper could be found in [27].

Proposition 1. The pair $L$ and $\bar{L}_{\Gamma}$ forms a Lie bialgebroid. The Lie algebroid differential $\bar{\partial}_{\Gamma}$ for the deformed Lie algebroid $\bar{L}_{\Gamma}$ acting on $L$ is given by $\bar{\partial}+\llbracket \Gamma,-\rrbracket$. i.e. for any section $\ell$ of $L$,

$$
\bar{\partial}_{\Gamma} \ell=\bar{\partial} \ell+\llbracket \Gamma, \ell \rrbracket .
$$


Proof. By definition, the vector bundle $\bar{L}_{\Gamma}$ is maximally isotropic in the Courant algebroid $\left(T \oplus T^{*}\right)_{\mathbb{C}}$. Since $\Gamma$ satisfies the Maurer-Cartan equation, it follows that the space of sections of $\bar{L}_{\Gamma}$ is closed with respect to the Courant bracket. To find a more precise description, we follow the computation in [21].

For $\bar{\sigma} \in C^{\infty}(\bar{L}), \ell_{1}, \ell_{2} \in C^{\infty}(L)$, define a Lie derivative by

$$
\left(\mathcal{L}_{\ell_{1}} \bar{\sigma}\right) \ell_{2}=\rho\left(\ell_{1}\right)\left(\bar{\sigma}\left(\ell_{2}\right)\right)-\bar{\sigma}\left(\llbracket \ell_{1}, \ell_{2} \rrbracket\right),
$$

where $\rho$ is the natural projection from $\left(T \oplus T^{*}\right)_{\mathbb{C}}$ onto $T_{\mathbb{C}}$. The property of the Lie derivative in algebroid theory could be found in [22]. Follow [21, Identity (23)], for any $\bar{\ell}_{1}, \bar{\ell}_{2} \in C^{\infty}(\bar{L})$ define

$$
\llbracket \bar{\ell}_{1}, \bar{\ell}_{2} \rrbracket_{\Gamma}=\mathcal{L}_{\Gamma \bar{\ell}_{1}} \bar{\ell}_{2}-\mathcal{L}_{\Gamma \bar{\ell}_{2}} \bar{\ell}_{2}+\bar{\partial}\left(\bar{\ell}_{1}\left(\Gamma \bar{\ell}_{2}\right)\right) .
$$

As noted in the proof of [21, Theorem 6.1], $\Gamma$ satisfies the Maurer-Cartan equation if and only if

$$
\llbracket \bar{\ell}_{1}+\Gamma \bar{\ell}_{1}, \bar{\ell}_{2}+\Gamma \bar{\ell}_{2} \rrbracket=\llbracket \bar{\ell}_{1}, \bar{\ell}_{2} \rrbracket+\llbracket \bar{\ell}_{1}, \bar{\ell}_{2} \rrbracket_{\Gamma}+\Gamma\left(\llbracket \bar{\ell}_{1}, \bar{\ell}_{2} \rrbracket+\llbracket \bar{\ell}_{1}, \bar{\ell}_{2} \rrbracket_{\Gamma}\right) .
$$

Now we are ready to compute the Lie algebroid differential of $\bar{L}_{\Gamma}$ with $L$ as its dual. For every $\ell \in C^{\infty}(L)$ and $\ell_{1}, \ell_{2} \in C^{\infty}(\bar{L})$,

$$
\begin{aligned}
& \left(\bar{\partial}_{\Gamma} \ell\right)\left(\bar{\ell}_{1}+\Gamma \bar{\ell}_{1}, \bar{\ell}_{2}+\Gamma \bar{\ell}_{2}\right) \\
= & \rho\left(\bar{\ell}_{1}+\Gamma \bar{\ell}_{1}\right)\left(\ell\left(\bar{\ell}_{2}\right)\right)-\rho\left(\bar{\ell}_{2}+\Gamma \bar{\ell}_{2}\right)\left(\ell\left(\bar{\ell}_{1}\right)\right)-\ell\left(\llbracket \bar{\ell}_{1}+\Gamma \bar{\ell}_{1}, \bar{\ell}_{2}+\Gamma \bar{\ell}_{2} \rrbracket\right) \\
= & \rho\left(\bar{\ell}_{1}+\Gamma \bar{\ell}_{1}\right)\left(\ell\left(\bar{\ell}_{2}\right)\right)-\rho\left(\bar{\ell}_{2}+\Gamma \bar{\ell}_{2}\right)\left(\ell\left(\bar{\ell}_{1}\right)\right) \\
& -\ell\left(\llbracket \bar{\ell}_{1}, \bar{\ell}_{2} \rrbracket+\llbracket \bar{\ell}_{1}, \bar{\ell}_{2} \rrbracket \Gamma+\Gamma\left(\llbracket \bar{\ell}_{1}, \bar{\ell}_{2} \rrbracket+\llbracket \bar{\ell}_{1}, \bar{\ell}_{2} \rrbracket \Gamma\right)\right) .
\end{aligned}
$$

Since the image of a section in $\bar{L}$ under $\Gamma$ is a section of $L$ and $L$ isotropic, the above is equal to

$$
\begin{aligned}
& =\rho\left(\bar{\ell}_{1}+\Gamma \bar{\ell}_{1}\right)\left(\ell\left(\bar{\ell}_{2}\right)\right)-\rho\left(\bar{\ell}_{2}+\Gamma \bar{\ell}_{2}\right)\left(\ell\left(\bar{\ell}_{1}\right)\right)-\ell\left(\left(\llbracket \bar{\ell}_{1}, \bar{\ell}_{2} \rrbracket+\llbracket \bar{\ell}_{1}, \bar{\ell}_{2} \rrbracket_{\Gamma}\right)\right) \\
& =(\bar{\partial} \ell)\left(\bar{\ell}_{1}, \bar{\ell}_{2}\right)+\rho\left(\Gamma \bar{\ell}_{1}\right)\left(\ell\left(\bar{\ell}_{2}\right)\right)-\rho\left(\Gamma \bar{\ell}_{2}\right)\left(\ell\left(\bar{\ell}_{1}\right)\right)-\ell\left(\llbracket \bar{\ell}_{1}, \bar{\ell}_{2} \rrbracket_{\Gamma}\right) .
\end{aligned}
$$

The proof of this proposition is completed if we could show that

$$
\llbracket \Gamma, \ell \rrbracket\left(\bar{\ell}_{1}, \bar{\ell}_{2}\right)=\rho\left(\Gamma \bar{\ell}_{1}\right)\left(\ell\left(\bar{\ell}_{2}\right)\right)-\rho\left(\Gamma \bar{\ell}_{2}\right)\left(\ell\left(\bar{\ell}_{1}\right)\right)-\ell\left(\llbracket \bar{\ell}_{1}, \bar{\ell}_{2} \rrbracket_{\Gamma}\right) .
$$

It is now a matter of definition of Lie derivative to show that the right hand side of the above is equal to

$$
\bar{\ell}_{2}\left(\llbracket \Gamma \bar{\ell}_{1}, \ell \rrbracket\right)-\bar{\ell}_{1}\left(\llbracket \Gamma \bar{\ell}_{2}, \ell \rrbracket\right)-\rho(\ell)\left(\bar{\ell}_{1}\left(\Gamma \bar{\ell}_{2}\right)\right) .
$$

Finally, the following identity always hold for any section $\Gamma$ of $\wedge^{2} L, \ell$ of $L$ and $\bar{\ell}_{1}, \bar{\ell}_{2}$ of $\bar{L}$.

$$
\llbracket \Gamma, \ell \rrbracket\left(\bar{\ell}_{1}, \bar{\ell}_{2}\right)=\bar{\ell}_{2}\left(\llbracket \Gamma \bar{\ell}_{1}, \ell \rrbracket\right)-\bar{\ell}_{1}\left(\llbracket \Gamma \bar{\ell}_{2}, \ell \rrbracket\right)-\rho(\ell)\left(\bar{\ell}_{1}\left(\Gamma \bar{\ell}_{2}\right)\right)
$$

See [27] for a detailed proof for (18). Therefore, the proof of the proposition is completed.

As far as analyzing the deformation of associated differential Gerstenhaber algebras is concerned, the above observation reduces an analysis to one on deformation of differentials. As the bundle structure could remain constant, we now focus on the 
variation from $\bar{\partial}$ to $\bar{\partial}_{\Gamma}$, and hence denote the respective differential Gerstenhaber algebras by $\operatorname{DGA}(\bar{\partial})$ and $\operatorname{DGA}\left(\bar{\partial}_{\Gamma}\right)$.

To compare DGA $\left(\bar{\partial}_{\Gamma}\right)$ with DGA $(\bar{\partial})$, we identify the constraints for them to be homomorphic. If the homomorphism induces an isomorphism at cohomology level, these two DGAs are said to be quasi-isomorphic. In such case, the generalized complex structure $J$ and the deformed one are also said to form a weak mirror pair [24], [6], [7]. Let

$$
\Phi: L \rightarrow L
$$

be a vector bundle homomorphism depending on $\Gamma$. It induces a homomorphism of the exterior algebra generated by $L$. That is

$$
\Phi(A \wedge B):=\Phi(A) \wedge \Phi(B)
$$

for any $A, B \in C^{\infty}\left(\wedge^{\bullet} L\right)$.

It is a homomorphism of graded Lie algebras if for any $A, B$ in $C^{\infty}\left(\wedge^{\bullet} L\right)$,

$$
\llbracket \Phi(A), \Phi(B) \rrbracket=\Phi(\llbracket A, B \rrbracket) .
$$

If in addition, when the following diagram is commutative for all $0 \leq k \leq n$,

$$
\begin{array}{ccc}
\wedge^{k} L & \stackrel{\bar{\partial}_{\Gamma}}{\longrightarrow} & \wedge^{k+1} L \\
\Phi \downarrow & & \downarrow \Phi \\
\wedge^{k} L & \stackrel{\bar{\partial}}{\longrightarrow} & \wedge^{k+1} L,
\end{array}
$$

we have a homomorphism of differential Gerstenhaber algebras

$$
\Phi:\left(\wedge^{\bullet} L, \llbracket-,-\rrbracket, \wedge, \bar{\partial}_{\Gamma}\right) \rightarrow\left(\wedge^{\bullet} L, \llbracket-,-\rrbracket, \wedge, \bar{\partial}\right) .
$$

In general, if the bundle homomorphism intertwines the differentials as in (21) and satisfies (19), then the map $\Phi$ is an exterior differential algebra homomorphism. If the bundle homomorphism intertwines the differentials as in (21) and satisfies (20), the map $\Phi$ is a graded differential algebra homomorphism.

The diagram (21) above is equivalent to

$$
\Phi \circ \bar{\partial}_{\Gamma}=\bar{\partial} \circ \Phi .
$$

By Proposition 3.1, that means for any section $A$ of the bundle $\wedge^{\bullet} L$,

$$
\Phi(\bar{\partial} A+\llbracket \Gamma, A \rrbracket)=\bar{\partial}(\Phi A) .
$$

Suppose that $\Omega$ is a closed 2 -form and $L$ is a generalized complex structure, then

$$
\bar{L}_{\Omega}=\left\{X+\alpha+\iota_{X} \Omega: X+\alpha \in \bar{L}\right\}
$$

is again a generalized complex structure. This is because the closedness of $\Omega$ and its skew-symmetry imply that the map

$$
X+\alpha \mapsto X+\alpha+\iota_{X} \Omega
$$

is an automorphism of the Courant bracket $\llbracket-,-\rrbracket$ in (5) and the non-degenerate bilinear pairing $\langle-,-\rangle$ in (3). This map is known as a $B$-field transformation by the 
closed 2-form $\Omega$. It naturally defines an isomorphism between the DGA of the bundle $L$ and the DGA of the $B$-field transformation of $L$.

On infinitesimal level, deformation theory of generalized complex structures is elliptic and a copy of Kuranishi theory follows [17]. In the spirit of Kuranishi theory, let $\phi$ be the infinitesimal version of $\Phi$. It is now an endomorphism from the vector bundle $L$ to $L$. The infinitesimal version of (23) becomes

$$
\phi(\bar{\partial} A)+\llbracket \Gamma_{1}, A \rrbracket=\bar{\partial}(\phi A),
$$

where $\Gamma_{1}$ is the infinitesimal deformation, representing an element in the second cohomology $H_{J}^{2}$. The infinitesimal version of $(20)$ is

$$
\llbracket \phi A, B \rrbracket+\llbracket A, \phi B \rrbracket=\phi \llbracket A, B \rrbracket .
$$

In these expressions, the map $\phi$ is an infinitesimal version of a homomorphism of exterior algebras. Therefore, it is an endomorphism with following property.

$$
\phi(A \wedge B)=(\phi A) \wedge B+A \wedge(\phi B) .
$$

Now we treat $\phi$ as an element in $L^{*} \otimes L=\operatorname{End}(L)$. More generally, it is a section of $\operatorname{End}\left(\wedge^{n} L\right)$. On the other hand, $\Gamma_{1}$ is a section of $\wedge^{2} L$.

Now we summarize the above discussion with a concept and its implication to variation of the structure of associated differential Gerstenhaber algebras.

Definition 1. Suppose that $M$ is a manifold with a generalized complex structure $J$, whose $+i$-eigenbundle is $L$. A section $\Gamma_{1} \in C^{\infty}\left(\wedge^{2} L\right)$ and a section $\phi \in C^{\infty}\left(L^{*} \otimes L\right)$ form a compatible pair if $\bar{\partial} \Gamma_{1}=0$ and

$$
\begin{gathered}
\bar{\partial}(\phi A)-\phi(\bar{\partial} A)=\llbracket \Gamma_{1}, A \rrbracket ; \\
\llbracket \phi A, B \rrbracket+\llbracket A, \phi B \rrbracket=\phi \llbracket A, B \rrbracket ; \\
\phi(A \wedge B)=(\phi A) \wedge B+A \wedge(\phi B) .
\end{gathered}
$$

Note that if $\Gamma_{1}$ is in the center of the Gerstenhaber algebra, i.e. $\llbracket \Gamma_{1}, A \rrbracket=0$ for all $A \in C^{\infty}\left(\wedge^{\bullet} L\right)$, then $\phi=0$ is an obvious solution for the above three identities. For future reference, we note the following

Proposition 2. Suppose that $M$ is a manifold with a generalized complex structure $J$, whose $+i$-eigenbundle is $L$. Let $\Gamma_{1} \in C^{\infty}\left(\wedge^{2} L\right)$ be a closed section: $\bar{\partial} \Gamma_{1}=0$. Then $\left(\Gamma_{1}, \phi=0\right)$ form a compatible pair if and only if $\Gamma_{1}$ is central: $\llbracket \Gamma_{1}, A \rrbracket=0$ for all $A \in C^{\infty}\left(\wedge^{\bullet} L\right)$.

THEOREM 1. Suppose that $M$ is a manifold with a generalized complex structure $J$, whose $+i$-eigenbundle is $L$. Suppose that $\Gamma$ is an integrable deformation with infinitesimal deformation $\Gamma_{1}$. If there is a homomorphism $\Phi$ of the bundle $L$ to itself such that it generates a homomorphism from the differential Gerstenhaber algebra of the deformation generalized complex structure to the un-perturbed one, then there exists a compatible pair $\Gamma_{1}$ and $\phi$ such that $\bar{\partial} \Gamma_{1}=0$, and up to first order, $\Gamma$ is equal to $\Gamma_{1}$ and $\Phi$ is equal to $1+\phi$. 
3.2. Integrability of compatible pairs. Given a compatible pair $\Gamma_{1}$ and $\phi$, an immediate issue is whether they actually come from a deformation $\Gamma$ and a homomorphism of DGAs. In this section, we apply the principles of Kuranishi's recursive method to prove that this is the case. We will divide the proof of the following theorem in several steps.

THEOREM 2. Suppose that $M$ is a manifold with a generalized complex structure $J$, whose $+i$-eigenbundle is $L$. Let $\Gamma_{1} \in C^{\infty}\left(\wedge^{2} L\right)$ and $\phi \in C^{\infty}\left(L^{*} \otimes L\right)$ be a compatible pair. Let $t$ be a real variable. Define

$$
\Gamma(t)=\sum_{n=1}^{\infty}(-1)^{n-1} \frac{1}{n !} t^{n} \phi^{n-1} \Gamma_{1}, \quad \Phi(t)=\sum_{n=1}^{\infty} \frac{1}{n !} t^{n} \phi^{n} .
$$

Then $\Gamma(t)$ satisfies the Maurer-Cartan equation. Moveover, if $\operatorname{DGA}(\Gamma(t))$ represents the Differential Gerstenhaber algebra $\left(\wedge \bullet L, \llbracket-,-\rrbracket, \wedge, \bar{\partial}_{\Gamma(t)}\right)$, then $\Phi(t)$ is a homomorphism from $\operatorname{DGA}(\Gamma(t))$ to $\mathrm{DGA}(\Gamma(0))$.

The Maurer-Cartan equation at degree- 1 with respect to the variable $t$ is simply $\bar{\partial} \Gamma_{1}=0$. For $n \geq 2$, it is

$$
(-1)^{n-1} \frac{1}{n !} \bar{\partial}\left(\phi^{n-1} \Gamma_{1}\right)+\frac{1}{2} \sum_{j+k=n} \llbracket(-1)^{k-1} \frac{1}{k !} \phi^{k-1} \Gamma_{1},(-1)^{j-1} \frac{1}{j !} \phi^{j-1} \Gamma_{1} \rrbracket=0 .
$$

Let the binomial coefficients be

$$
C_{k}^{n}=\frac{n !}{k !(n-k) !} .
$$

Then the above equation is equivalent to

$$
\bar{\partial}\left(\phi^{n-1} \Gamma_{1}\right)=\frac{1}{2} \sum_{k=1}^{n-1} C_{k}^{n} \llbracket \phi^{k-1} \Gamma_{1}, \phi^{n-k-1} \Gamma_{1} \rrbracket .
$$

Similarly, $\Phi(t)$ is a homomorphism of $\llbracket-,-\rrbracket$ and $\wedge$ if and only if for degree $n$, and for any sections $A$ and $B$ of $L$,

(33) $\frac{1}{n !} \phi^{n} \llbracket A, B \rrbracket=\sum_{k+j=n} \llbracket \frac{1}{k !} \phi^{k} A, \frac{1}{j !} \phi^{j} B \rrbracket, \quad \frac{1}{n !} \phi^{n}(A \wedge B)=\sum_{k+j=n}\left(\frac{1}{k !} \phi^{k} A \wedge \frac{1}{j !} \phi^{j} B\right)$.

It is equivalent to

$$
\phi^{n} \llbracket A, B \rrbracket=\sum_{k=1}^{n} C_{k}^{n} \llbracket \phi^{k} A, \phi^{n-k} B \rrbracket, \quad \phi^{n}(A \wedge B)=\sum_{k=1}^{n} C_{k}^{n}\left(\phi^{k} A \wedge \phi^{n-k} B\right) .
$$

Finally, $\Phi(t)$ intertwines $\bar{\partial}_{\Gamma(t)}$ if and only if they satisfy the identity (23). Assuming that $\Phi(t)$ is a homomorphism of the Courant bracket on $\wedge^{\bullet} L$, we need to show that for any section $A$ of $\wedge^{\bullet} L$,

$$
\Phi(t)(\bar{\partial} A)+\llbracket \Phi(t) \Gamma(t), \Phi(t) A \rrbracket=\bar{\partial}(\Phi(t) A) .
$$

Consider the infinite product $\Phi(t) \Gamma(t)$. Its degree- $n$ term is equal to

$$
\sum_{k+j=n} \frac{1}{j !} \phi^{j}(-1)^{k-1} \frac{1}{k !} \phi^{k-1} \Gamma_{1}=\left(\sum_{k+j=n}(-1)^{k-1} \frac{1}{j ! k !}\right) \phi^{n-1} \Gamma_{1} .
$$


On the other hand, consider the power series

$$
g=\sum_{k=1}^{\infty}(-1)^{k} \frac{1}{k !} x^{k-1} \quad \text { and } \quad e^{x}=\sum_{k=1}^{\infty} \frac{1}{k !} x^{k} .
$$

We have $1-x g=e^{-x}$. Therefore, $e^{x}-x e^{x} g=1$. i.e. $e^{x}(x g)=e^{x}-1$. Equating the $n$-th order terms for $n \geq 1$, we find that

$$
\sum_{k+j=n}(-1)^{k-1} \frac{1}{j ! k !}=\frac{1}{n !} .
$$

Therefore, $\Phi(t) \Gamma(t)=\sum_{n \geq 1} \frac{1}{n !} t^{n} \phi^{n-1} \Gamma_{1}$. Then the identity (35) at degree $n$ becomes

$$
\frac{1}{n !} \phi^{n} \bar{\partial} A+\sum_{k+j=n} \llbracket \frac{1}{k !} \phi^{k-1} \Gamma_{1}, \frac{1}{j !} \phi^{j} A \rrbracket=\frac{1}{n !} \bar{\partial} \phi^{n} A .
$$

Equivalently, it is

$$
\bar{\partial} \phi^{n} A-\phi^{n} \bar{\partial} A=\sum_{k=1}^{n} C_{k}^{n} \llbracket \phi^{k-1} \Gamma_{1}, \phi^{n-k} A \rrbracket .
$$

To complete a proof of Theorem 2, we need to prove that the identities (32), (34) and (36) hold.

Lemma 1. Suppose that $\Gamma_{1}$ and $\phi$ form a compatible pair, then identity (32) holds.

Proof. Using the fact that $\bar{\partial} \Gamma_{1}=0$ and equation (27), we get a telescopic sum

$$
\begin{aligned}
\bar{\partial} \phi^{n-1} \Gamma_{1} & =\bar{\partial}\left(\phi^{n-1} \Gamma_{1}\right)-\phi \bar{\partial} \phi^{n-2} \Gamma_{1} \\
& +\phi \bar{\partial} \phi^{n-2} \Gamma_{1}-\phi^{2} \bar{\partial} \phi^{n-3} \Gamma_{1}+\phi^{2} \bar{\partial} \phi^{n-3} \Gamma_{1}-\phi^{3} \bar{\partial} \phi^{n-4} \Gamma_{1} \\
& +\ldots \quad \cdots \quad \cdots+\phi^{n-2} \bar{\partial} \phi \Gamma_{1}-\phi^{n-1} \bar{\partial} \Gamma_{1} \\
& =\sum_{h=0}^{n-2} \phi^{h} \llbracket \Gamma_{1}, \phi^{n-2-h} \Gamma_{1} \rrbracket .
\end{aligned}
$$

Since $\phi$ satisfies (28) and the Schouten bracket is commutative when restricted to section of $\Lambda^{2} L$, we rewrite the above identity as

$$
\begin{aligned}
\bar{\partial} \phi^{n-1} \Gamma_{1} & =\sum_{h=0}^{n-2} \sum_{k=0}^{h} C_{k}^{h} \llbracket \phi^{k} \Gamma_{1}, \phi^{n-2-k} \Gamma_{1} \rrbracket=\sum_{h=1}^{n-1} \sum_{k=1}^{h} C_{k-1}^{h-1} \llbracket \phi^{k-1} \Gamma_{1}, \phi^{n-1-k} \Gamma_{1} \rrbracket \\
& =\sum_{k=1}^{n-1}\left(\sum_{h=k}^{n-1} C_{k-1}^{h-1}\right) \llbracket \phi^{k-1} \Gamma_{1}, \phi^{n-1-k} \Gamma_{1} \rrbracket \\
& =\sum_{k=1}^{n-1} C_{k}^{n-1} \llbracket \phi^{k-1} \Gamma_{1}, \phi^{n-1-k} \Gamma_{1} \rrbracket .
\end{aligned}
$$

Performing the index substitution $k \mapsto n-k$ and using the commutativity of the Schouten bracket again, we get

$$
\begin{aligned}
\bar{\partial} \phi^{n-1} \Gamma_{1} & =\frac{1}{2} \sum_{k=1}^{n-1}\left(C_{k}^{n-1}+C_{k-1}^{n-1}\right) \llbracket \phi^{k-1} \Gamma_{1}, \phi^{n-1-k} \Gamma_{1} \rrbracket \\
& =\frac{1}{2} \sum_{k=1}^{n-1} C_{k}^{n} \llbracket \phi^{k-1} \Gamma_{1}, \phi^{n-1-k} \Gamma_{1} \rrbracket .
\end{aligned}
$$


Lemma 2. Suppose that $\Gamma_{1}$ and $\phi$ form a compatible pair, then the two identities in (34) hold.

Proof. It is an elementary induction. The proof for both cases are identical. We work only through the case with Schouten bracket. When $n=1$, the equation (34) is precisely the equation (28), which is satisfied by assumption. Assuming that the equation (34) holds for all $k \leq n$. We next compute $\phi^{n+1} \llbracket A, B \rrbracket$, which we take as $\phi\left(\phi^{n} \llbracket A, B \rrbracket\right)$. By induction hypothesis, it is equal to

$$
\begin{aligned}
& \sum_{k=0}^{n} C_{k}^{n} \phi\left(\llbracket \phi^{n-k} A, \phi^{k} B \rrbracket\right) \\
= & \sum_{k=0}^{n} C_{k}^{n}\left(\llbracket \phi^{n+1-k} A, \phi^{k} B \rrbracket+\llbracket \phi^{n-k} A, \phi^{k+1} B \rrbracket\right) \\
= & \llbracket \phi^{n+1} A, B \rrbracket+\sum_{k=1}^{n} C_{k}^{n} \llbracket \phi^{n+1-k} A, \phi^{k} B \rrbracket \\
& +\sum_{k=0}^{n-1} C_{k}^{n} \llbracket \phi^{n-k} A, \phi^{k+1} B \rrbracket+\llbracket A, \phi^{n+1} B \rrbracket \\
= & \llbracket \phi^{n+1} A, B \rrbracket+\sum_{k=1}^{n}\left(C_{k}^{n}+C_{k-1}^{n}\right) \llbracket \phi^{n+1-k} A, \phi^{k} B \rrbracket+\llbracket A, \phi^{n+1} B \rrbracket \\
= & \sum_{k=0}^{n+1} C_{k}^{n+1} \llbracket \phi^{n-k} A, \phi^{k} B \rrbracket .
\end{aligned}
$$

Lemma 3. Suppose that $\Gamma_{1}$ and $\phi$ form a compatible pair, then identity (36) holds for all $n \geq 1$.

Proof. Since $\bar{\partial} \Gamma_{1}=0$, we substitute $A$ by $\Gamma_{1}$ in (27) to see that identity (36) holds when $n=1$. Assume that (36) holds for all $k \leq n$. We next prove that it holds for $n+1$. Since

$$
\begin{aligned}
& \bar{\partial} \phi^{n+1} A-\phi^{n+1} \bar{\partial} A \\
= & \bar{\partial} \phi^{n}(\phi A)-\phi^{n}(\bar{\partial} \phi A)+\phi^{n}((\bar{\partial} \phi A)-\phi \bar{\partial} A),
\end{aligned}
$$

by induction hypothesis, the above is equal to

$$
\sum_{k=1}^{n} C_{k}^{n} \llbracket \phi^{k-1} \Gamma_{1}, \phi^{n+1-k} A \rrbracket+\phi^{n} \llbracket \Gamma_{1}, A \rrbracket .
$$

By Lemma 2, it is equal to

$$
\begin{aligned}
& \sum_{k=1}^{n} C_{k}^{n} \llbracket \phi^{k-1} \Gamma_{1}, \phi^{n+1-k} A \rrbracket+\sum_{k=0}^{n} C_{k}^{n} \llbracket \phi^{k} \Gamma_{1}, \phi^{n-k} A \rrbracket \\
= & \sum_{k=1}^{n}\left(C_{k}^{n}+C_{k-1}^{n}\right) \llbracket \phi^{k-1} \Gamma_{1}, \phi^{n+1-k} A \rrbracket+\llbracket \phi^{n} \Gamma_{1}, A \rrbracket .
\end{aligned}
$$


By Pascal Identity, it is equal to

$$
\sum_{k=1}^{n} C_{k}^{n+1} \llbracket \phi^{k-1} \Gamma_{1}, \phi^{n+1-k} A \rrbracket+\llbracket \phi^{n} \Gamma_{1}, A \rrbracket=\sum_{k=1}^{n+1} C_{k}^{n+1} \llbracket \phi^{k-1} \Gamma_{1}, \phi^{n+1-k} A \rrbracket .
$$

4. Holomorphic Poisson manifolds. On a complex manifold $(M, J), L=$ $T^{1,0} \oplus T^{*(0,1)}$ and $\bar{L}=L^{*}=T^{0,1} \oplus T^{*(1,0)}$. Therefore, the exterior bundle has a decomposition

$$
\wedge^{\bullet} L=\oplus_{k}\left(\oplus_{p+q=k} \wedge^{p} T^{1,0} \otimes \wedge^{q} T^{*(0,1)}\right) .
$$

We will use the notations $T^{p, 0}=\wedge^{p} T^{1,0}$ and $T^{*(0, q)}=\wedge^{q} T^{*(0,1)}$. Sections of $T^{p, 0}$ are addressed as $(p, 0)$-vectors, more generally polyvector fields.

4.1. Type decomposition of deformations. The cohomology of DGA $(J)$ decomposes into the direct sum of classical Dolbeault cohomology with the sheaf of exterior product of the holomorphic tangent bundle as coefficients.

$$
H_{J}^{k}=\oplus_{p+q=k} H^{q}\left(M, T^{p, 0}\right) .
$$

If $\Gamma_{1}$ is in $H_{J}^{2}$ it has three components:

$$
\Gamma_{1}=\Lambda+\widehat{\Gamma}_{1}+\Omega \in H^{0}\left(M, T^{2,0}\right) \oplus H^{1}\left(M, T^{1,0}\right) \oplus H^{2}(M, \mathcal{O}),
$$

where $\Lambda$ is a $(2,0)$-bivector field, $\Omega$ is a $(0,2)$-form, and $\widehat{\Gamma}_{1}$ is a classical infinitesimal complex deformation. Similarly,

$$
\begin{aligned}
L^{*} \otimes L= & \operatorname{End}(L, L) \\
= & \operatorname{End}\left(T^{1,0}, T^{1,0}\right) \oplus \operatorname{End}\left(T^{*(0,1)}, T^{*(0,1)}\right) \\
& \quad \oplus \operatorname{End}\left(T^{1,0}, T^{*(0,1)}\right) \oplus \operatorname{End}\left(T^{*(0,1)}, T^{1,0}\right) .
\end{aligned}
$$

If $\phi$ is a section of $L^{*} \otimes L$, we represent its decomposition by $\phi=\phi_{1}+\phi_{2}+\phi_{3}+\phi_{4}$ such that

$$
\begin{aligned}
& \phi_{1} \in C^{\infty}\left(\operatorname{End}\left(T^{1,0}, T^{1,0}\right)\right), \quad \phi_{2} \in C^{\infty}\left(\operatorname{End}\left(T^{*(0,1)}, T^{*(0,1)}\right)\right), \\
& \phi_{3} \in C^{\infty}\left(\operatorname{End}\left(T^{1,0}, T^{*(0,1)}\right)\right), \quad \phi_{4} \in C^{\infty}\left(\operatorname{End}\left(T^{*(0,1)}, T^{1,0}\right)\right) .
\end{aligned}
$$

Proposition 3. A pair $\Gamma_{1} \in C^{\infty}\left(M, \wedge^{2} L\right)$ and $\phi \in C^{\infty}\left(M, L^{*} \otimes L\right)$ is compatible if and only if the pairs $\left(\Lambda, \phi_{4}\right),\left(\Omega, \phi_{3}\right)$ and $\left(\widehat{\Gamma}_{1}, \phi_{1}+\phi_{2}\right)$ are compatible.

Proof. This proposition is an inspection of type decompositions. For example,

$$
\bar{\partial} \Gamma_{1}=\bar{\partial} \Lambda+\bar{\partial} \widehat{\Gamma}_{1}+\bar{\partial} \Omega .
$$

Since $\bar{\partial} \Lambda \in C^{\infty}\left(M, T^{2,0} \otimes T^{*(0,1)}\right), \bar{\partial} \widehat{\Gamma}_{1} \in C^{\infty}\left(M, T^{1,0} \otimes T^{*(0,2)}\right)$, and $\bar{\partial} \Omega \in$ $C^{\infty}\left(M, T^{*(0,3)}\right)$, each component has to vanish individually if $\bar{\partial} \Gamma_{1}=0$. i.e.

$$
\bar{\partial} \Lambda=0, \quad \bar{\partial} \widehat{\Gamma}_{1}=0, \quad \bar{\partial} \Omega=0 .
$$


Next, for all $Z \in C^{\infty}\left(M, T^{1,0}\right)$ and $\bar{\omega} \in C^{\infty}\left(M, T^{*(0,1)}\right)$,

$$
\begin{gathered}
\llbracket \Lambda, Z \rrbracket \in C^{\infty}\left(T^{2,0}\right), \quad \llbracket \Lambda, \bar{\omega} \rrbracket \in C^{\infty}\left(T^{(1,0)} \otimes T^{*(0,1)}\right) ; \\
\llbracket \widehat{\Gamma}_{1}, Z \rrbracket \in C^{\infty}\left(T^{*(0,2)}\right), \quad \llbracket \widehat{\Gamma}_{1}, \bar{\omega} \rrbracket=0 ; \\
\llbracket \Omega, Z \rrbracket \in C^{\infty}\left(T^{1,0} \otimes T^{*(0,1)}\right), \quad \llbracket \Omega, \bar{\omega} \rrbracket \in C^{\infty}\left(T^{*(0,2)}\right) .
\end{gathered}
$$

On the other hand,

$$
\begin{gathered}
\bar{\partial}\left(\phi_{1}(Z)\right)-\phi_{1}(\bar{\partial} Z) \in C^{\infty}\left(T^{1,0} \otimes T^{*(0,1)}\right), \quad \bar{\partial}\left(\phi_{1}(\bar{\omega})\right)-\phi_{1}(\bar{\partial} \bar{\omega})=0, \\
\bar{\partial}\left(\phi_{2}(Z)\right)-\phi_{2}(\bar{\partial} Z)=-\phi_{2}(\bar{\partial} Z) \in C^{\infty}\left(T^{1,0} \otimes T^{*(0,1)}\right), \quad \bar{\partial}\left(\phi_{2}(\bar{\omega})\right)-\phi_{2}(\bar{\partial} \bar{\omega}) \in C^{\infty}\left(T^{*(0,2)}\right), \\
\bar{\partial}\left(\phi_{3}(Z)\right)-\phi_{3}(\bar{\partial} Z) \in C^{\infty}\left(T^{*(0,2)}\right), \quad \bar{\partial}\left(\phi_{3}(\bar{\omega})\right)-\phi_{3}(\bar{\partial} \bar{\omega})=0 \\
\bar{\partial}\left(\phi_{4}(Z)\right)-\phi_{4}(\bar{\partial} Z)=-\phi_{4}(\bar{\partial} Z) \in C^{\infty}\left(T^{2,0}\right), \quad \bar{\partial}\left(\phi_{4}(\bar{\omega})\right)-\phi_{4}(\bar{\partial} \bar{\omega}) \in C^{\infty}\left(T^{1,0} \otimes T^{*(0,1)}\right) .
\end{gathered}
$$

By equating the types, we arrive at the conclusion of this proposition.

In view of the last proposition and the decomposition of $H_{J}^{2}$, one should focus an initial analysis of deformations on the simple types, namely those whose infinitesimal deformations are contained in a unique summand of the decomposition of $H_{J}^{2}$.

Infinitesimal deformations given by a $\bar{\partial}$-closed section $\widehat{\Gamma}_{1}$ of $T^{1,0} \otimes T^{*(0,1)}$ could always be represented and analyzed as classical complex deformation theory.

If one considers a $\bar{\partial}$-closed 2 -form representing an element in $H^{2}(M, \mathcal{O})$, then by definition of Courant bracket $\llbracket \Omega, \Omega \rrbracket=0$. Therefore, $\Omega$ satisfies the Maurer-Cartan equation, and

$$
L_{\bar{\Omega}}=\left\{X+\alpha+\iota_{X} \bar{\Omega}: X+\alpha \in L\right\}
$$

is a generalized complex structure. The issue of integrability is trivial. However, this deformation does not change the type of the generalized complex structure. It is still type- $n$ where $n$ is the complex dimension of the manifold $M$. If the $(0,2)$-form $\Omega$ is not only $\bar{\partial}$-closed but also closed, then this deformation is trivial within the realm of generalized complex structures because the deformation is only the result of a B-field transformation [17].

4.2. Holomorphic bivector fields. Suppose that $\Gamma$ is a deformation whose first order term is a bivector field $\Lambda$ with $\bar{\partial} \Lambda=0$. Let $\Gamma_{2}$ be its second order term. As $\Gamma$ satisfies the Maurer-Cartan equation, up to second order term, we have

$$
\bar{\partial}\left(t \Lambda+t^{2} \Gamma_{2}\right)+\frac{1}{2} \llbracket t \Lambda+t^{2} \Gamma_{2}, t \Lambda+t^{2} \Gamma_{2} \rrbracket=0 .
$$

It yields

$$
\bar{\partial} \Gamma_{2}+\frac{1}{2} \llbracket \Lambda, \Lambda \rrbracket=0 .
$$

Since $\Lambda$ is a bivector, $\llbracket \Lambda, \Lambda \rrbracket$ is a $(3,0)$-vector field. On the other hand, $\bar{\partial} \Gamma_{2}$ must have a components with $(0,1)$-forms. Therefore, the only solution is when $\llbracket \Lambda, \Lambda \rrbracket=0$. It follows immediately that $\Lambda$ is a solution of the Maurer-Cartan equation and $\Gamma_{2}$ could be chosen to be zero. Therefore, a bivector field $\Lambda$ representing an element in $H^{0}\left(M, T^{2,0}\right)$ is an infinitesimal deformation of an integrable deformation if and only if $\llbracket \Lambda, \Lambda \rrbracket=0$.

Definition 2. A $(2,0)$-vector field is a holomorphic Poisson structure on a complex manifold if $\bar{\partial} \Lambda=0$ and $\llbracket \Lambda, \Lambda \rrbracket=0$. In such case, we call $\Lambda$ a holomorphic Poisson vector field. 
Given such a $\Lambda$, suppose that $\phi \in C^{\infty}\left(\operatorname{End}\left(T^{*(0,1)}, T^{1,0}\right)\right)$ is compatible with $\Lambda$. By Theorem 2, $\Phi=\sum \frac{1}{n !} \phi^{n}$ is a DGA homomorphism. However, as an endomorphism from the bundle $L$ to $L$, its kernel contains at least $T^{1,0}$. Therefore, $\phi \Lambda=0$ and $\phi \circ \phi=0$. Therefore, we could conclude that the homomorphism $\Phi$ is simply $1+\phi$. Furthermore, given a section $X+\bar{\alpha}$ of $T^{1,0} \oplus T^{*(0,1)}, \Phi(X+\bar{\alpha})=X+\phi(\bar{\alpha})+\bar{\alpha}$. As the vector part is $X+\phi(\bar{\alpha})$ and the form part is $\bar{\alpha}, X+\bar{\alpha}$ is in the kernel of $\Phi$ if and only if it is identically zero. Therefore, $\Phi$ as a bundle map from $L$ to $L$ is an isomorphism. It is extended to an isomorphism from the exterior bundle $\wedge^{\bullet} L$ to $\wedge^{\bullet} L$. Therefore, $\Phi$ is not only a DGA homomorphism, but also an isomorphism. We summarize our observation below.

THEOREM 3. Let $M$ be a complex manifold with a holomorphic Poisson vector field $\Lambda$. Suppose that $\phi$ is a section of $\operatorname{End}\left(T^{*(0,1)}, T^{1,0}\right)$ compatible with the $\Lambda$ in the sense of Definition 1. Then $\Lambda$ defines a family of generalized complex deformation of the complex structure on $M$ with $t \Lambda$. Moreover, if $\mathrm{DGA}(t \Lambda)$ represents the $\mathrm{DGA}$ of the deformed complex structure, then they are all isomorphic to DGA(0), the differential Gerstenhaber algebra of the complex structure on the manifold $M$.

Although from the viewpoint of deformation of DGAs, the presence of a compatible pair on a holomorphic Poisson manifold makes the deformation of DGAs trivial, on the geometric level, it is non-trivial. Recall that

$$
L_{\bar{\Lambda}}=\left\{X+\bar{\alpha}+\iota \bar{\alpha} \bar{\Lambda}: X+\bar{\alpha} \in T^{1,0} \oplus T^{*(0,1)}\right\} .
$$

As $\iota \bar{\alpha} \bar{\Lambda}$ is a $(0,1)$-vector, the type of the generalized complex structure $L_{\bar{\Lambda}}$ is different from the un-deformed one $L$. If $\Lambda$ as a bundle map from $T^{*(0,1)}$ to $T^{1,0}$ is everywhere non-degenerate, then $L_{\bar{\Lambda}}$ is a type- 0 generalized complex structure. By a Gualtieri's lemma [17], there exists a symplectic structure $\Omega$ on the manifold $M$ such that the complexified DGA of $\omega$ is isomorphic to that of $\operatorname{DGA}(\Lambda)$ via a B-field transformation. Since $\operatorname{DGA}(\Lambda)$ is isomorphic to $\operatorname{DGA}(0)$. We obtain the following result.

THEOREM 4. Let $M$ be a manifold with complex structure $J$. Denote its associated DGA by $\operatorname{DGA}(J)$. Suppose that $\Lambda$ is a non-degenerate holomorphic Poisson structure. If there exists a section of $T^{1,0} \otimes T^{0,1}$ compatible with $\Lambda$ in the sense of Definition 1 , then there exists a symplectic structure $\Omega$ in the deformation family of $J$ such that $\operatorname{DGA}(\Omega)$ is isomorphic to $\operatorname{DGA}(J)$.

In the sense of Merkulov, the pair $(M, J)$ and $(M, \Omega)$ form a weak mirror pair [6] [7] [24].

4.3. Rational surfaces. In this section, we compute the first cohomology of some well known holomorphic Poisson manifolds to demonstrate that for many holomorphic Poisson structures, Theorem 3 does not have solution.

Assume that we have a compact holomorphic Poisson manifold. Denote the Poisson bivector field by $\Lambda$. Consider $Z$ a section of $T^{1,0}$ and $\bar{\omega}$ a section of $T^{*(0,1)}$. Then $Z+\bar{\omega}$ is a section of $L=T^{1,0} \oplus T^{*(0,1)}$. By Proposition 1, it represents an element of the first cohomology of DGA $\left(\bar{\partial}_{\Lambda}\right)$ if and only if $\bar{\partial}_{\Lambda}(Z+\bar{\omega})=0$. That is

$$
\begin{aligned}
\bar{\partial}_{\Lambda}(Z+\bar{\omega}) & =\bar{\partial} Z+\llbracket \Lambda, Z \rrbracket+\bar{\partial} \bar{\omega}+\llbracket \Lambda, \bar{\omega} \rrbracket \\
& =\llbracket \Lambda, Z \rrbracket+\bar{\partial} Z+\llbracket \Lambda, \bar{\omega} \rrbracket+\bar{\partial} \bar{\omega}=0 .
\end{aligned}
$$

The terms above are sections of $\wedge^{2} L=T^{2,0} \oplus T^{1,0} \otimes T^{*(0,1)} \oplus T^{*(0,2)}$. As each component in this decomposition has to vanish, we conclude that

$$
\llbracket \Lambda, Z \rrbracket=0, \quad \bar{\partial} Z+\llbracket \Lambda, \bar{\omega} \rrbracket=0, \quad \bar{\partial} \bar{\omega}=0 .
$$


In particular the $(0,1)$-form $\bar{\omega}$ is $\bar{\partial}$-closed. To push this computation further, assume that the Dolbeault cohomology $H^{1}(M, \mathcal{O})$ vanishes. It follows that the $(0,1)$-form is $\bar{\partial}$-exact, and there is a smooth function $f$ on the manifold $M$ such that $\bar{\omega}=\bar{\partial} f$. Consider the vector field $V=\llbracket \Lambda, f \rrbracket$. Since $\bar{\partial} \Lambda=0$,

$$
\bar{\partial} V=\bar{\partial} \llbracket \Lambda, f \rrbracket=\llbracket \bar{\partial} \Lambda, f \rrbracket-\llbracket \Lambda, \bar{\partial} f \rrbracket=-\llbracket \Lambda, \bar{\omega} \rrbracket .
$$

With (39) above, we conclude that $\bar{\partial}(Z-V)=0$. Therefore, $Z-V$ is a holomorphic vector field on the manifold $M$. By Jacobi identity of Gerstenhaber algebras,

$$
\llbracket \Lambda, \llbracket \Lambda, f \rrbracket \rrbracket+\llbracket \Lambda, \llbracket f, \Lambda \rrbracket \rrbracket+\llbracket f, \llbracket \Lambda, \Lambda \rrbracket \rrbracket=0 .
$$

Since $\llbracket \Lambda, \Lambda \rrbracket=0$, the above is reduced to

$$
\llbracket \Lambda, V \rrbracket=\llbracket \Lambda, \llbracket \Lambda, f \rrbracket \rrbracket=0 .
$$

Combined with the first identity in (39), we conclude that

$$
\llbracket \Lambda, Z-V \rrbracket=0 .
$$

Let $W=Z-V$, then $Z=W+V=W+\llbracket \Lambda, f \rrbracket$ such that $\bar{\partial} W=0$ and $\llbracket \Lambda, W \rrbracket=0$. Moreover, the section

$$
Z+\bar{\omega}=W+\llbracket \Lambda, f \rrbracket+\bar{\partial} f=W+\bar{\partial}_{\Lambda} f .
$$

Since $\bar{\partial}_{\Lambda} f$ is $\bar{\partial}_{\Lambda}$-exact, $W$ and $Z+\bar{\omega}$ represent the same cohomology class in $H \bar{\partial}_{\Lambda}$.

Proposition 4. Suppose that $M$ is a holomorphic Poisson manifold with Poisson vector field $\Lambda$. If $H^{1}(M, \mathcal{O})$ vanishes, then

$$
H \frac{1}{\partial_{\Lambda}}=\left\{W \in H^{0}\left(M, T^{1,0}\right): \llbracket \Lambda, W \rrbracket=0\right\} .
$$

On the other hand, the first cohomology of DGA $(\bar{\partial})$ is equal to

$$
H^{0}\left(M, T^{1,0}\right) \oplus H^{1}(M, \mathcal{O}) .
$$

Given the assumption of Proposition 4, it is equal to $H^{0}\left(M, T^{1,0}\right)$. It is easy to find example on which there exists non-trivial holomorphic Poisson structures but it does not admit compatible pairs due to the difference between $H^{0}\left(M, T^{1,0}\right)$ and $H_{\bar{\partial}_{\Lambda}}$. For instance, there is a classification of compact complex surfaces admitting holomorphic Poisson structures [3]. Among them, the minimal rational surfaces are all holomorphic Poisson manifolds with vanishing irregularity. Except when the surface is a complex projective plane, they are rational ruled surfaces.

For the complex projective plane $\Lambda$ is an element in $H^{0}\left(\mathbf{C P}^{2}, \mathcal{O}(3)\right)$. It could be identified to a homogeneous polynomial of degree- 3 in the homogeneous coordinates of the complex projective plane. Meanwhile the space of holomorphic vector fields $H^{0}\left(\mathbf{C P}^{2}, T^{1,0}\right)$ is the complex algebra $\mathfrak{s l}(3, \mathbb{C})$, treated as the set of $3 \times 3$-matrices acting on of $\mathbb{C}^{3}$ by natural matrix multiplications. From this perspective, for any $W$ in $H^{0}\left(\mathbf{C P}^{2}, T^{1,0}\right)$, the action $\llbracket W,-\rrbracket$ on $H^{0}\left(\mathbf{C P}^{2}, \mathcal{O}(3)\right)$ is the induced representation of $\mathfrak{s l}(3, \mathbb{C})$ on the third symmetric product $S^{3} \mathbb{C}^{3}$. Then for each $\Lambda \neq 0$, one could find a $W$ such that $\llbracket W, \Lambda \rrbracket \neq 0$. Therefore, for each holomorphic Poisson structure on the complex projective plane, $H_{\bar{\partial}_{\Lambda}}$ is strictly smaller then $H \frac{1}{\partial}=H^{0}\left(\mathbf{C P}^{2}, T^{1,0}\right)=$ $\mathfrak{s l}(3, \mathbb{C})$. It shows that $\mathrm{DGA}\left(\mathbf{C P}^{2}, \bar{\partial}\right)$ and $\operatorname{DGA}\left(\mathbf{C P}^{2}, \bar{\partial}_{\Lambda}\right)$ for any holomorphic Poisson structure could never be quasi-isomorphic. 
4.4. Hopf surfaces. In this section, we compute $H_{\bar{\partial}_{\Lambda}}$ when the underlying manifold $M$ is the Hopf surface, and demonstrates that it does admit compatible pairs.

Consider $\mathbb{C}^{2}$ with coordinates $z=\left(z_{1}, z_{2}\right)$. Let $\lambda>1$ be a real number. It generates a one-parameter group of automorphism on $\mathbb{C}^{2}$. The quotient of $\mathbb{C}^{2} \backslash\{0\}$ with respect this group is diffeomorphic to the Lie group $M=U(1) \times S U(2)$. The complex structure on $\mathbb{C}^{2}$ descends onto $M$ to define an integrable complex structure, invariant of the left-action of the Lie group. In this section, by Hopf surface, we mean this particular complex structure. The classical complex deformation theory of this complex structure was analyzed by Dabrowski [12]. We focus on the deformations generated by its holomorphic Poisson structures. Consider

$$
\begin{aligned}
X_{0} & =\frac{1}{2}\left(z_{1} \frac{\partial}{\partial z_{1}}+z_{2} \frac{\partial}{\partial z_{2}}\right), & X_{1} & =\frac{i}{2}\left(z_{1} \frac{\partial}{\partial z_{1}}-z_{2} \frac{\partial}{\partial z_{2}}\right) \\
X_{2} & =\frac{i}{2}\left(z_{2} \frac{\partial}{\partial z_{1}}+z_{1} \frac{\partial}{\partial z_{2}}\right), & X_{3} & =\frac{1}{2}\left(-z_{2} \frac{\partial}{\partial z_{1}}+z_{1} \frac{\partial}{\partial z_{2}}\right),
\end{aligned}
$$

and

$$
\bar{\sigma}=\bar{\partial} \ln |z|^{2}=\frac{z_{1} d \bar{z}_{1}+z_{2} d \bar{z}_{2}}{\left|z_{1}\right|^{2}+\left|z_{2}\right|^{2}} .
$$

The cohomology spaces for the $\operatorname{DGA}(J)$ are given below. The computation of these cohomology spaces are not new. We do not present any details.

$$
\begin{gathered}
H^{1}(M, \mathcal{O})=\langle\bar{\sigma}\rangle, \quad H^{0}\left(M, T^{1,0}\right)=\left\langle X_{0}, X_{1}, X_{2}, X_{3}\right\rangle \cong \mathfrak{u}(1) \oplus \mathfrak{s l}(2), \\
H^{1}\left(M, T^{1,0}\right)=\left\langle X_{0} \wedge \bar{\sigma}, X_{1} \wedge \bar{\sigma}, X_{2} \wedge \bar{\sigma}, X_{3} \wedge \bar{\sigma}\right\rangle \\
H^{0}\left(M, T^{2,0}\right)=\left\langle X_{0} \wedge X_{1}, X_{0} \wedge X_{2}, X_{0} \wedge X_{3}\right\rangle \\
H^{1}\left(M, T^{2,0}\right)=\left\langle X_{0} \wedge X_{1} \wedge \bar{\sigma}, X_{0} \wedge X_{2} \wedge \bar{\sigma}, X_{0} \wedge X_{3} \wedge \bar{\sigma}\right\rangle .
\end{gathered}
$$

In addition,

$$
\begin{gathered}
\llbracket X_{0}, X_{1} \rrbracket=0, \quad \llbracket X_{0}, X_{2} \rrbracket=0, \quad \llbracket X_{0}, X_{3} \rrbracket=0, \\
\llbracket X_{1}, X_{2} \rrbracket=-X_{3}, \quad \llbracket X_{2}, X_{3} \rrbracket=-X_{1}, \quad \llbracket X_{3}, X_{1} \rrbracket=-X_{2} .
\end{gathered}
$$

Set $f=\ln |z|^{2}$, then $\mathcal{L}_{X_{0}} f=\frac{1}{2}$. For $j=1,2,3$, define $f_{j}=\mathcal{L}_{X_{j}} f$, then

$$
f_{1}=\frac{i}{2|z|^{2}}\left(z_{1} \bar{z}_{1}-z_{2} \bar{z}_{2}\right), \quad f_{2}=\frac{i}{2|z|^{2}}\left(z_{2} \bar{z}_{1}+z_{1} \bar{z}_{2}\right), \quad f_{3}=\frac{1}{2|z|^{2}}\left(-z_{2} \bar{z}_{1}+z_{1} \bar{z}_{2}\right) .
$$

The functions $f_{1}, f_{2}, f_{3}$ are invariant of the group of actions generated by $\left(\lambda z_{1}, \lambda z_{2}\right)$, and hence they are globally defined on the quotient space $M$. Then we have

$$
\llbracket X_{0}, \bar{\sigma} \rrbracket=0, \quad \llbracket X_{1}, \bar{\sigma} \rrbracket=\bar{\partial} f_{1}, \quad \llbracket X_{2}, \bar{\sigma} \rrbracket=\bar{\partial} f_{2}, \quad \llbracket X_{3}, \bar{\sigma} \rrbracket=\bar{\partial} f_{3} .
$$

Whenever $A=a_{1} X_{1}+a_{2} X_{2}+a_{3} X_{3}$ is a holomorphic vector field in the $\mathfrak{s l}(2)$ component of $H^{0}\left(M, T^{1,0}\right)$,

$$
\llbracket A, \bar{\sigma} \rrbracket=a_{1} \llbracket X_{1}, \bar{\sigma} \rrbracket+a_{2} \llbracket X_{2}, \bar{\sigma} \rrbracket+a_{3} \llbracket X_{3}, \bar{\sigma} \rrbracket=\bar{\partial}\left(a_{1} f_{1}+a_{2} f_{2}+a_{3} f_{3}\right) .
$$

We use the notation $f_{A}$ to denote the function $a_{1} f_{1}+a_{2} f_{2}+a_{3} f_{3}$. By (46),

$$
\llbracket A, \bar{\sigma} \rrbracket=\bar{\partial} f_{A} .
$$


Since $X_{0}$ commutes with $X_{j}$ for $j=1,2,3, \mathcal{L}_{X_{0}} f_{j}=\mathcal{L}_{X_{j}} \mathcal{L}_{X_{0}} f=\mathcal{L}_{X_{j}} \frac{1}{2}=0$. Then for all $A$

$$
\mathcal{L}_{X_{0}} f_{A}=0
$$

Given the above preparation, we begin to compute the first cohomology of $\operatorname{DGA}\left(\bar{\partial}_{\Lambda}\right)$ where $\Lambda$ is any holomorphic Poisson structure on $M$. Let $A=a_{1} X_{1}+$ $a_{2} X_{2}+a_{3} X_{3}$ be a holomorphic vector field, then $\Lambda=X_{0} \wedge A$ is a holomorphic Poisson structure. As noted in (42), by choosing the complex numbers $\left(a_{1}, a_{2}, a_{3}\right)$, we exhaust all holomorphic Poisson structure.

Now we calculate the first cohomology with respect to $\bar{\partial}_{\Lambda}=\bar{\partial}+\llbracket \Lambda,-\rrbracket$. Suppose that $Z$ is a smooth $(1,0)$-vector field and $\bar{\omega}$ is a smooth $(0,1)$-form. $\bar{\partial}_{\Lambda}(Z+\bar{\omega})=0$ if and only if $Z$ and $\bar{\omega}$ satisfy the constraints (39). Once again, they are

$$
\bar{\partial} \bar{\omega}=0, \quad \llbracket \Lambda, Z \rrbracket=0, \quad \bar{\partial} Z+\llbracket \Lambda, \bar{\omega} \rrbracket=0 .
$$

Since the cohomology $H^{1}(X, \mathcal{O})$ is spanned by $\bar{\sigma}$, there exists a function $\psi$ and a unique complex number $a$ such that

$$
\bar{\omega}=a \bar{\sigma}+\bar{\partial} \psi
$$

Let $V$ be the vector field $\llbracket \Lambda, \psi \rrbracket$. Since $\bar{\partial} \Lambda=0$,

$$
\begin{aligned}
\bar{\partial} V & =\bar{\partial} \llbracket \Lambda, \psi \rrbracket=\llbracket \bar{\partial} \Lambda, \psi \rrbracket-\llbracket \Lambda, \bar{\partial} \psi \rrbracket=-\llbracket \Lambda, \bar{\partial} \psi \rrbracket \\
& =-\llbracket \Lambda, \bar{\omega}-a \bar{\sigma} \rrbracket=-\llbracket \Lambda, \bar{\omega} \rrbracket+a \llbracket \Lambda, \bar{\sigma} \rrbracket .
\end{aligned}
$$

By definition of $\Lambda,(46)$ and (47), this is equal to

$$
-\llbracket \Lambda, \bar{\omega} \rrbracket+a X_{0} \wedge \llbracket A, \bar{\sigma} \rrbracket=-\llbracket \Lambda, \bar{\omega} \rrbracket+a X_{0} \wedge \bar{\partial} f_{A}=-\llbracket \Lambda, \bar{\omega} \rrbracket-a \bar{\partial}\left(f_{A} X_{0}\right) .
$$

It follows from (49) that

$$
\bar{\partial}\left(V-Z+a f_{A} X_{0}\right)=0 .
$$

Next, consider the Schouten bracket. By (49),

$$
\begin{aligned}
& \llbracket \Lambda, V-Z+a f_{A} X_{0} \rrbracket \\
= & \llbracket \Lambda, \llbracket \Lambda, \psi \rrbracket \rrbracket-\llbracket \Lambda, Z \rrbracket+\llbracket X_{0} \wedge A, a f_{A} X_{0} \rrbracket \\
= & \llbracket \Lambda, \llbracket \Lambda, \psi \rrbracket \rrbracket+a X_{0} \wedge \llbracket A, f_{A} X_{0} \rrbracket-a A \wedge \llbracket X_{0}, f_{A} X_{0} \rrbracket \\
= & \llbracket \Lambda, \llbracket \Lambda, \psi \rrbracket \rrbracket+a X_{0} \wedge \llbracket A, f_{A} \rrbracket X_{0}+a f_{A} X_{0} \wedge \llbracket A, X_{0} \rrbracket-a A \wedge \llbracket X_{0}, f_{A} \rrbracket X_{0} .
\end{aligned}
$$

Due to (44) and (48), this is equal to $\llbracket \Lambda, \llbracket \Lambda, \psi \rrbracket \rrbracket$. By the Jacobi identity for Gerstenhaber algebra and the fact that $\llbracket \Lambda, \Lambda \rrbracket=0, \llbracket \Lambda, V-Z+a f_{A} X_{0} \rrbracket=0$. Define

$$
W=-V+Z-a f_{A} X_{0}=-\llbracket X_{0} \wedge A, \psi \rrbracket+Z-a f_{A} X_{0} .
$$

Then $\llbracket \Lambda, W \rrbracket=0$. However, by (49) and the identity above,

$$
\llbracket \Lambda, W \rrbracket=\llbracket X_{0} \wedge A, W \rrbracket=X_{0} \wedge \llbracket A, W \rrbracket .
$$

As $\bar{\partial} W=0$, it is a linear combination of $X_{0}, X_{1}, X_{2}, X_{3}$. Therefore, $\llbracket \Lambda, W \rrbracket$ is equal to zero if and only if there exist constants $b$ and $c$ such that $W=b X_{0}+c A$. Therefore,

$$
Z=V+W+a f_{A} X_{0}=\llbracket \Lambda, \psi \rrbracket+b X_{0}+c A+a f_{A} X_{0} .
$$


As we have already resolved the first two constraints in (49), we could now substitute $Z$ in the last constraint to check that it does not generate additional conditions. So, $Z+\bar{\omega}$ is $\bar{\partial}_{\Lambda}$-closed for $\Lambda=X_{0} \wedge A$ if and only if there exist a function $\psi$ and constants $a, b, c$ such that

$$
\begin{aligned}
& \bar{\omega}=a \bar{\sigma}+\bar{\partial} \psi, \\
& Z=\llbracket \Lambda, \psi \rrbracket+b X_{0}+c A+a f_{A} X_{0}=\llbracket \Lambda, \psi \rrbracket+W+a f_{A} X_{0} .
\end{aligned}
$$

Since

$$
Z+\bar{\omega}=\llbracket \Lambda, \psi \rrbracket+b X_{0}+c A+a f_{A} X_{0}+a \bar{\sigma}+\bar{\partial} \psi=b X_{0}+c A+a f_{A} X_{0}+a \bar{\sigma}+\bar{\partial}_{\Lambda} \psi,
$$

$Z+\bar{\omega}$ and $b X_{0}+c A+a f_{A} X_{0}+a \bar{\sigma}$ represent the same element in the first cohomology space $H^{1}\left(M, \bar{\partial}_{X_{0} \wedge A}\right)$. Therefore, we have

$$
H^{1}\left(X, \bar{\partial}_{X_{0} \wedge A}\right)=\left\langle X_{0}, A, f_{A} X_{0}+\bar{\sigma}\right\rangle \cong \mathbb{C}^{3} .
$$

On the other hand, it is noted in (40) that the first cohomology of $\operatorname{DGA}(J)$ is a five-dimensional space.

$$
H^{1}(M, \bar{\partial})=H^{1}(M, \mathcal{O}) \oplus H^{0}\left(M, T^{1,0}\right)=\langle\bar{\sigma}\rangle \oplus\left\langle X_{0}, X_{1}, X_{2}, X_{3}\right\rangle .
$$

Therefore, along the deformation given by holomorphic Poisson vector field $\Lambda=X_{0} \wedge$ $A$, the first cohomology jumps and hence $\Lambda$ could not be part of any compatible pair.

5. Holomorphic symplectic algebras. In an explicit computation in [26], part of the result in Theorem 4 has been observed on the Kodaira-Thurston surface. It was possible to do an explicit computation due to the fact that the manifold is a low-dimension nilmanifold.

If $H$ is a simply-connected nilpotent Lie group and $K$ is a co-compact subgroup, then the quotient manifold $M=H / K$ is said to be a nilmanifold. Let $\mathfrak{h}$ be the Lie algebra of the group $H$, the Chevalley-Eilenberg differential $d$ determines a complex

$$
d: \wedge^{k} \mathfrak{h}^{*} \rightarrow \wedge^{k+1} \mathfrak{h}^{*}
$$

It is known for a long time that the inclusion $\mathfrak{h}^{*}$ as invariant section of $T^{*}$ induces an isomorphism on the cohomology level [25]. If the nilmanifold has an invariant symplectic structure $\Omega$, one could therefore consider this inclusion as a quasi-isomorphism from the differential Gerstenhaber algebra with invariant objects $\operatorname{DGA}(\mathfrak{h}, \Omega)$ to the manifold level DGA $(H / K, \Omega)$.

There were a series of attempt to attain a similar result for Dolbeault cohomology [8] [9] [10] [28]. This body of research generates a collection of examples of nilmanifolds for which the inclusion of invariant sections in the space of sections of the bundle $L=T^{1,0} \oplus T^{*(0,1)}$ induces a quasi-isomorphism of DGAs. Kodaira-Thurston surfaces is a prominent example with small dimension. To illustrate the theory of the past few chapters, we now focus on $\operatorname{DGA}(\mathfrak{h}, J)$ for some Lie algebra $\mathfrak{h}$.

In our subsequent computation, we do not restrict $\mathfrak{h}$ to being nilpotent, but will construct algebras on which there is a good collection of geometric objects as in [7].

5.1. Pseudo-Kähler structures. Let $(\mathfrak{g}, \omega)$ denote a real Lie algebra equipped with a symplectic structure $\omega$. Let $V$ denote the underlying vector space of $\mathfrak{g}$. We seek a linear map $\gamma: \mathfrak{g} \rightarrow \operatorname{End}(V)$ such that for all $x, y, z \in \mathfrak{g}$,

$$
\begin{aligned}
& \gamma(x) y-\gamma(y) x=[x, y] \\
& \omega(\gamma(x) y, z)+\omega(y, \gamma(x) z)=0 \\
& \gamma([x, y])=\gamma(x) \gamma(y)-\gamma(y) \gamma(x)
\end{aligned}
$$


The last condition requires $\gamma$ to be a representation. The second condition means that it is a symplectic representation.

If one uses $\gamma$ as a operator of vector fields on the Lie group of the algebra $\mathfrak{g}$, the last condition is equivalent to require $\gamma$ to be a flat connection. Condition in (56) is to require the connection to be symplectic. The condition in (55) is to require the connection to be torsion-free.

Given the representation $\gamma$, one obtains a semi-direct product Lie algebra $\mathfrak{h}:=$ $\mathfrak{g} \ltimes V$ with a Lie bracket defined by

$$
\llbracket(x, 0),(y, 0) \rrbracket=([x, y], 0) \quad \llbracket(x, 0),(0, v) \rrbracket=(0, \gamma(x) v),
$$

for all $x, y \in \mathfrak{g}$ and $v \in V$. Here we denote a generic element in $\mathfrak{g} \ltimes V$ in terms of the decomposition $(x, u) \in \mathfrak{g} \oplus V$.

On the semi-direct product, consider the linear map.

$$
J(x, y)=(-y, x) .
$$

This is an almost complex structure. The $(1,0)$ vectors are given by

$$
\mathfrak{h}^{1,0}=\left\{(x,-i x) \in(\mathfrak{g} \oplus V)_{\mathbb{C}}: x \in \mathfrak{g}\right\} .
$$

$J$ is an integrable complex structure due to (55) because

$$
\llbracket x-i J x, y-i J y \rrbracket=\llbracket(x,-i x),(y,-i y) \rrbracket=([x, y],-i(\gamma(x) y-\gamma(y) x)) .
$$

The symplectic structure $\omega$ induces three different symplectic forms on the semi-direct product $\mathfrak{h}$.

$$
\begin{gathered}
\Omega_{1}((x, u),(y, v)):=-\omega(x, v)-\omega(u, y), \\
\Omega_{2}((x, u),(y, v)):=\omega(x, y)-\omega(u, v), \\
\Omega_{3}((x, u),(y, v)):=\omega(x, y)+\omega(u, v) .
\end{gathered}
$$

With respect to the complex structure $J, \Omega_{c}=\Omega_{1}+i \Omega_{2}$ is a closed (2,0)-form. It is non-degenerate in the sense that the contraction map

$$
V \mapsto \Omega_{c}(V,), \quad \Omega_{c}: \mathfrak{h}^{1,0} \rightarrow \mathfrak{h}^{*(1,0)}
$$

is non-degenerate. The pair $\left(\Omega_{c}, J\right)$ is called a complex symplectic structure on the algebra $\mathfrak{h}$. Let $\Lambda$ be the inverse mapping of $\Omega_{c}$.

$$
\Lambda: \mathfrak{h}^{*(1,0)} \rightarrow \mathfrak{h}^{1,0} .
$$

It is a matter of definition that $\Lambda \in \wedge^{2} \mathfrak{h}^{1,0}=\mathfrak{h}^{2,0}$. Therefore, it could play the role of an invariant holomorphic Poisson structure. Indeed we have the following

Lemma 4. Let $\Lambda$ be the inverse of $\Omega_{c}$, then it satisfies the following.

- For any $\alpha, \beta \in \mathfrak{h}^{*(1,0)}, \Lambda(\alpha, \beta)=-\Omega_{c}\left(\Omega_{c}^{-1}(\alpha), \Omega_{c}^{-1}(\beta)\right)$.

- $\llbracket \Lambda, \Lambda \rrbracket=0$.

- $\bar{\partial} \Lambda=0$.

Proof. Beyond tracing definitions, the first identity is an elementary application of the algebraic properties of Gerstenhaber algebra. The second identity is equivalent to $d \Omega_{c}=0$. The last is another application of the algebraic properties of Gerstenhaber algebra combined with a type decomposition argument. 
The last lemma leads to the next.

LEMMA 5. Given a symplectic algebra $(\mathfrak{g}, \omega)$ with a flat torsion-free symplectic connection on the underlying vector space $V$ of $\mathfrak{g}$, then the semi-direct product $\mathfrak{h}=$ $\mathfrak{g} \ltimes V$ has a holomorphic Poisson structure $\left(J, \Lambda=\Omega_{c}^{-1}\right)$.

Given the above holomorphic Poisson structure, we consider the generalized deformation generated by the holomorphic Poisson vector field $\Lambda$. It yields

$$
L_{\bar{\Lambda}}=\mathfrak{h}^{1,0} \oplus\left\{\bar{\zeta}+\overline{\Lambda \zeta}: \bar{\zeta} \in \mathfrak{h}^{*(0,1)}\right\}
$$

Since $\bar{\Lambda}: \mathfrak{h}^{*(0,1)} \rightarrow \mathfrak{h}^{1,0}$ is an isomorphism with $\bar{\Omega}_{c}$ as its inverse,

$$
L_{\bar{\Lambda}}=\mathfrak{h}^{1,0} \oplus\left\{\bar{\Omega}_{c}(\bar{Y})+\bar{Y}: \bar{Y} \in \mathfrak{h}^{(0,1)}\right\} .
$$

Since $\bar{\Omega}_{c}$ is a $(0,2)$-form, for any $(1,0)$-vector $X, \bar{\Omega}_{c}(X)=0$. Therefore, the above is equal to

$$
\begin{aligned}
& =\left\{X+\bar{\Omega}_{c}(X)+\bar{Y}+\bar{\Omega}_{c}(\bar{Y}): X \in \mathfrak{h}^{1,0}, \bar{Y} \in \mathfrak{h}^{0,1}\right\} \\
& =\left\{V+\Omega_{c}(V): V \in \mathfrak{h}^{1,0} \oplus \mathfrak{h}^{0,1}\right\}=\left\{V+\Omega_{1}(V)-i \Omega_{2}(V): V \in \mathfrak{h}_{c}\right\} \\
& =e^{\Omega_{1}}\left\{V-i \Omega_{2}(V): V \in \mathfrak{h}_{c}\right\} .
\end{aligned}
$$

The last equality means that the deformed generalized complex structure $L_{\bar{\Lambda}}$ is the $B$-field transformation by the closed 2 -form $\Omega_{1}$ of the generalized complex structure defined by the symplectic form $\Omega_{2}$. In conclusion, we have

Proposition 5. Given a symplectic algebra $(\mathfrak{g}, \omega)$ with a flat torsion-free symplectic connection on the underlying vector space $V$ of $\mathfrak{g}$, then up to the B-field transformation with respect to the closed 2-form $\Omega_{1}$, the generalized deformation of the classical complex structure by holomorphic Poisson structure $\Lambda=\left(\Omega_{1}+i \Omega_{2}\right)^{-1}$ is the the symplectic structure $\Omega_{2}$. In particular, $\operatorname{DGA}\left(L_{\bar{\Lambda}}\right)$ is isomorphic to $\operatorname{DGA}\left(\Omega_{2}\right)$.

5.2. Compatible pairs. A different perspective in understanding $\operatorname{DGA}\left(L_{\bar{\Lambda}}\right)$ is in terms of compatible pair. That is to identify an element $\phi$ in $\mathfrak{h}^{0,1} \otimes \mathfrak{h}^{1,0}$ so that $(\Lambda, \phi)$ forms a compatible pair.

As $\Omega_{3}$ is a $(1,1)$-form and its contraction map is non-degenerate

$$
\Omega_{3}: \mathfrak{h}^{1,0} \rightarrow \mathfrak{h}^{*(0,1)},
$$

its inverse map

$$
\Omega_{3}^{-1}: \mathfrak{h}^{*(0,1)} \rightarrow \mathfrak{h}^{1,0}
$$

is a natural candidate to form a compatible pair with $\Lambda$.

On the other hand, if $g$ is a non-degenerate symmetric bilinear form the algebra $\mathfrak{g}$, it induces a non-degenerate form on $\mathfrak{g} \ltimes V$ by

$$
\Delta((x, u),(y, v))=g(x, y)+g(u, v)
$$

Then its fundamental form is a $(1,1)$-form:

$$
\Omega_{4}((x, u),(y, v))=\Delta(J(x, u),(y, v))=\Delta((-u, x),(y, v))=g(x, v)-g(y, u) .
$$


Therefore, $\Omega_{4}^{-1}$ is also a candidate to match with $\Lambda$ as a compatible pair. It is a natural question to ask when $\Omega_{4}$ is closed. It amounts to asking the pair $J$ and $\Delta$ to form a pseudo-Kähler structure.

LEMMA 6. The pair $(J, \Delta)$ on $\mathfrak{h}$ forms a pseudo-Kähler structure if and only if

$$
g(\gamma(x) y, w)-g(\gamma(y) x, w)-g(x, \gamma(y) w)+g(y, \gamma(x) w)=0
$$

for all $x, y, w \in \mathfrak{g}$.

Proof. For any $(x, u),(y, v),(z, w) \in \mathfrak{g} \ltimes V$, expand $d \Omega_{4}((x, u),(y, v),(z, w))$. Since $\gamma$ is torsion-free, it is equal to

$$
\begin{aligned}
& -g(\gamma(x) y, w)+g(\gamma(y) x, w)+g(x, \gamma(y) w)-g(y, \gamma(x) w) \\
& -g(\gamma(z) x, v)+g(\gamma(x) z, v)+g(z, \gamma(x) v)-g(x, \gamma(z) v) \\
& -g(\gamma(y) z, u)+g(\gamma(z) y, u)+g(y, \gamma(z) u)-g(z, \gamma(y) u) .
\end{aligned}
$$

Since the last three lines are cyclic permutations of $(x, u),(y, v)$ and $(z, w)$, if one of these lines is equal to zero, all three equal to zero and therefore $d \Omega_{4}=0$. Conversely, if $d \Omega_{4}=0$, set $z=u=v=0$. Then the last two lines equal to zero, and the lemma follows.

Suppose that $\left(\Omega_{c}, J\right)$ is a holomorphic symplectic structure on the semi-direct product $\mathfrak{h}=\mathfrak{g} \ltimes V$ as above. Let $\Omega_{3}$ and $\Delta$ be the natural symplectic and pseudometric structure on $\mathfrak{h}$. Assume that $(\Delta, J)$ is pseudo-Kähler. Both $\Omega_{3}^{-1}$ and $\Omega_{4}^{-1}$ are candidates to be compatible with $\Lambda=\Omega_{c}^{-1}$, so are their linear combinations. Below is a key technical result in this section.

Proposition 6. Suppose that $\left(\Omega_{c}, J\right)$ is a holomorphic symplectic structure on the semi-direct product $\mathfrak{h}=\mathfrak{g} \ltimes V$ as above. Let $\Omega_{3}$ and $\Delta$ be the natural symplectic and (pseudo-)metric structure on $\mathfrak{h}$. Assume that $(\Delta, J)$ is pseudo-Kähler structure. If there is a real number $\mu$ such that

$$
\left(g^{-1} \omega\right)(\gamma(a) b)=-4 \mu \gamma\left(\left(g^{-1} \omega\right)(a)\right)\left(\left(g^{-1} \omega\right)(b)\right)
$$

for all $a, b \in \mathfrak{g}$, then

$$
\phi=-\frac{i}{4} \Omega_{3}^{-1}+\mu \Omega_{4}^{-1}
$$

and $\Lambda=\Omega_{c}^{-1}$ forms a compatible pair.

In the expression (65), we consider the contractions with $\omega$ and $g$ as maps from the underlying vector space $V$ of $\mathfrak{g}$ to its dual. Therefore, $\jmath=g^{-1} \omega$ is a map from $V$ to $V$. The following are used frequently in our proof of Proposition 6 above.

Lemma 7. Recall that $\mathfrak{h}^{1,0}=\left\{(a,-i a) \in(\mathfrak{g} \oplus V)_{\mathbb{C}}: a \in \mathfrak{g}\right\}$.

- As $(0,1)$-forms, $\Omega_{3}((a,-i a),-)=-i \Omega_{4}((\jmath(a),-i \jmath(a)),-)$.

- As (1,0)-forms, $\Omega_{3}((a, i a),-)=i \Omega_{4}((\jmath(a), i \jmath(a)),-)$.

- As (1,0)-forms, $\Omega_{c}((a,-i a),-)=-2 \Omega_{4}((\jmath(a), i \jmath(a)),-)$.

- $\llbracket(a,-i a),(b, i b) \rrbracket^{1,0}=(-\gamma(b) a, i \gamma(b) a)$.

- $\llbracket(a,-i a),(b, i b) \rrbracket^{0,1}=(\gamma(a) b, i \gamma(a) b)$.

To prove Proposition 6 , we consider a generic linear combination of $\Omega_{3}^{-1}$ and $\Omega_{4}^{-1}$, $\phi=\lambda \Omega_{3}^{-1}+\mu \Omega_{4}^{-1}$. 
Note that we first extend both $\Omega_{3}^{-1}$ and $\Omega_{4}^{-1}$ by zeroes on $\mathfrak{h}^{1,0}$. Then they are extended as endomorphisms defined on $\mathfrak{h}^{1,0} \oplus \mathfrak{h}^{*(0,1)}$ to endomorphisms defined on the exterior product $\wedge^{\bullet}\left(\mathfrak{h}^{1,0} \oplus \mathfrak{h}^{*(0,1)}\right)$ through the identity (29), by linearity $\phi$ also satisfies (29). Therefore, we will determine the coefficients $\lambda$ and $\mu$ by solving the non-trivial constraints in (27) and (28).

In the current context, the constraint (27) is equivalent to requiring that for all $\ell_{1}, \ell_{2} \in \mathfrak{h}^{1,0} \oplus \mathfrak{h}^{*(0,1)}$,

$$
\phi\left(\llbracket \ell_{1}, \ell_{2} \rrbracket\right)=\llbracket \phi \ell_{1}, \ell_{2} \rrbracket+\llbracket \ell_{1}, \phi \ell_{2} \rrbracket .
$$

Since $\mathfrak{h}^{1,0}$ is annihilated by $\phi$, and it is closed with respect to Schouten bracket, if both $\ell_{1}$ and $\ell_{2}$ are in $\mathfrak{h}^{1,0}$, then the identity (67) is trivially satisfied, and hence does not pose any constraint on $\lambda$ and $\mu$.

If $\ell_{1} \in \mathfrak{h}^{1,0}$, then there exists $a \in \mathfrak{g}$ such that $\ell_{1}=(a,-i a)$. If $\ell_{2} \in \mathfrak{h}^{*(0,1)}$, then there exists $(b,-i b) \in \mathfrak{h}^{1,0}$ such that $\ell_{2}=\Omega_{3}((b,-i b),-)$. By Lemma 7 ,

$$
\ell_{2}=\Omega_{3}((b,-i b),-)=\Omega_{4}((-i \jmath(b),-\jmath(b)),-) .
$$

Since $\phi \ell_{1}=0$, the constraint in $(67)$ is reduced to $\phi \llbracket \ell_{1}, \ell_{2} \rrbracket=\llbracket \ell_{1}, \phi \ell_{2} \rrbracket$. Since both sides of this identity are $(1,0)$-vectors, to verify that they are identical, it suffices to show that the evaluation of any $(1,0)$-forms on these two vectors are identical. Since $\Omega_{3}$ is non-degenerate, any $(1,0)$-form has the form $\Omega_{3}((n, i n),-)$ for some $(0,1)$-vector $(n, i n)$. Then a proof of $(67)$ is reduced to check whether the following holds:

$$
\Omega_{3}\left((n, i n), \phi \llbracket \ell_{1}, \ell_{2} \rrbracket\right)-\Omega_{3}\left((n, i n), \llbracket \ell_{1}, \phi \ell_{2} \rrbracket\right)=0 .
$$

Making use of various definitions and Lemma 7, we reduce the above identity to

$$
\begin{aligned}
& \lambda \Omega_{3}((b,-i b),(\gamma(a) \jmath(n),-\gamma(a) \jmath(n)))-\lambda \Omega_{3}((n, i n),([a, b],-i[a, b])) \\
& +\mu \Omega_{4}((-i \jmath(b),-\jmath(b)),(i \gamma \jmath(n),-\gamma(a) \jmath(n))) \\
& -\mu \Omega_{4}((i \jmath(n),-\jmath(n)),(-i[a, \jmath(b)],-[a, \jmath(b)]))=0 .
\end{aligned}
$$

Using definition of $\Omega_{3}$ and $\Omega_{4}$ in terms of $\omega$, the above is reduced to

$$
-\lambda \omega(\gamma(b) n, a)+i \mu g(\gamma(\jmath(b)) \jmath(n), a)=0 .
$$

It is equivalent to

$$
\lambda \jmath(\gamma(b) n)=i \mu \gamma(\jmath(b))(\jmath(n))
$$

for all $b, n \in \mathfrak{g}$. This identity is the first preliminary constraint on $\mu$ and $\lambda$.

Similarly, if $\ell_{1}, \ell_{2} \in \mathfrak{h}^{*(0,1)}$, choose $(a,-i a)$ and $(b,-i b)$ such that

$$
\ell_{1}=\Omega_{3}((a,-i a),-), \quad \ell_{2}=\Omega_{3}((b,-i b),-) .
$$

Since $\llbracket \ell_{1}, \ell_{2} \rrbracket=0,(67)$ is reduced to

$$
\llbracket \phi \ell_{1}, \ell_{2} \rrbracket+\llbracket \ell_{1}, \phi \ell_{2} \rrbracket=0 .
$$

As both terms in the above sum are $(0,1)$-forms, then its evaluation on any $(0,1)$-vector $(n, i n)$ is equal to zero. Substitute (69) into identity $(70)$, evaluate on a $(0,1)$-vector $(n, i n)$, and make use of Lemma 7 , we get

$$
\begin{aligned}
& -\lambda \Omega_{3}((b,-i b),(\gamma(a) n, i \gamma(a) n))+\lambda \Omega_{3}((a,-i a),(\gamma(b) n, i \gamma(b) n)) \\
& -\mu \Omega_{4}((-i \jmath(b),-\jmath(b)),(-i \gamma(\jmath(a)) n, \gamma(\jmath(a)) n)) \\
& +\mu \Omega_{4}((-i \jmath(a),-\jmath(a)),(-i \gamma(\jmath(b)) n, \gamma(\jmath(b)) n))=0 .
\end{aligned}
$$


Using definitions of $\Omega_{3}$ and $\Omega_{4}$, together with Lemma 7 , The above identity is reduced to

$$
\lambda \omega([a, b], n)-i \mu g([\jmath(a), \jmath(b)], n)
$$

for all $n \in \mathfrak{g}$. That is

$$
\lambda \jmath([a, b])=i \mu[\jmath(a), \jmath(b)] .
$$

Since $\gamma(a) b-\gamma(b) a=[a, b]$ for all $a, b$, the above is equivalent to

$$
\lambda \jmath(\gamma(a) b)-\lambda \jmath(\gamma(b) a)=-\mu \gamma(\jmath(a)) \jmath(b)+\mu \gamma(\jmath(b)) \jmath(a) .
$$

This identity holds for all $a, b \in \mathfrak{g}$ so long as (68) holds. Therefore, (68) is the only constraint for solving (67).

Next, we need to find the constraints on $\lambda$ and $\mu$ to satisfy the identify (28). This is equivalent to requiring

$$
\lambda\left(\bar{\partial} \Omega_{3}^{-1}(\ell)-\Omega_{3}^{-1} \bar{\partial} \ell\right)+\mu\left(\bar{\partial} \Omega_{4}^{-1}(\ell)-\Omega_{4}^{-1} \bar{\partial} \ell\right)=\llbracket \Lambda, \ell \rrbracket
$$

for all $\ell \in \mathfrak{h}^{1,0} \oplus \mathfrak{h}^{*(0,1)}$.

Since $\Omega_{3}^{-1}$ and $\Omega_{4}^{-1}$ are extended by zero on $\mathfrak{h}^{1,0}$, when $\ell$ is an element in $\mathfrak{h}^{1,0}$, the constraint $(72)$ is reduced to

$$
-\lambda \Omega_{3}^{-1} \bar{\partial} \ell-\mu \Omega_{4}^{-1} \bar{\partial} \ell=\llbracket \Lambda, \ell \rrbracket .
$$

Let $A, B$ be elements in $\mathfrak{h}^{1,0}$, with identity (18) and the fact that $d \Omega_{c}=0$, one could check that

$$
\llbracket \Lambda, \ell \rrbracket\left(\Omega_{c} A, \Omega_{c} B\right)=\Omega_{c}(\ell, \llbracket A, B \rrbracket) .
$$

If we set $\ell=(x,-i x), A=(a,-i a), B=(b,-i b)$ with $x, a, b \in \mathfrak{g}$, recall the definitions of $\Omega_{c}$ in terms of $\omega$, then the above is further simplified to

$$
\llbracket \Lambda, \ell \rrbracket\left(\Omega_{c} A, \Omega_{c} B\right)=4 i \omega(x,[a, b]) .
$$

In view of (11), the first term on the left-hand-side of the identity in (73) evaluated on the ordered pair $\Omega_{c} A, \Omega_{c} B$ is simplified to

$$
\begin{aligned}
& -\Omega_{3}^{-1} \bar{\partial} \ell\left(\Omega_{c} A, \Omega_{c} B\right) \\
= & -2 i\left(\Omega_{c} B(\llbracket(x,-i x),(a, i a) \rrbracket)-\Omega_{c} A(\llbracket(x,-i x),(b, i b) \rrbracket)\right. \\
= & -2 i\left(\Omega_{c}\left((b,-i b), \llbracket(x,-i x),(a, i a) \rrbracket^{1,0}\right)-\Omega_{c}\left((a,-i a), \llbracket(x,-i x),(b, i b) \rrbracket^{1,0}\right)\right) .
\end{aligned}
$$

With Lemma 7 and various definitions, one could show that

$$
-\Omega_{3}^{-1} \bar{\partial} \ell\left(\Omega_{c} A, \Omega_{c} B\right)=-8 \omega(x,[a, b]) .
$$

Similarly,

$$
\begin{aligned}
& -\Omega_{4}^{-1} \bar{\partial} \ell\left(\Omega_{c} A, \Omega_{c} B\right) \\
= & -2\left(\Omega_{c} A(-\gamma(\jmath b) x, i \gamma(\jmath b) x)-\Omega_{c} B(-\gamma(\jmath a) x, i \gamma(\jmath a) x)\right) .
\end{aligned}
$$

By Lemma 7 , it is equal to

$$
2\left(\Omega_{4}((-2 \jmath a,-2 i \jmath a),(-\gamma(\jmath b) x, i \gamma(\jmath b) x))-\Omega_{4}((-2 \jmath b,-2 i \jmath b),(-\gamma(\jmath a) x, i \gamma(\jmath a) x))\right) .
$$


By definition of $\Omega_{4}$, we have

$$
-\Omega_{4}^{-1} \bar{\partial} \ell\left(\Omega_{c} A, \Omega_{c} B\right)=8 g(i[\jmath a, \jmath b], x) .
$$

Since (68) is satisfied, (71) holds. Therefore,

$$
-\mu \Omega_{4}^{-1} \bar{\partial} \ell\left(\Omega_{c} A, \Omega_{c} B\right)=8 \lambda g(\jmath[a, b], x)=-8 \lambda \omega(x,[a, b]) .
$$

Combined the above identity with (75) and (74), we obtain

$$
-16 \lambda \omega(x,[a, b])=4 i \omega(x,[a, b])
$$

for all $x, a, b \in \mathfrak{g}$. Therefore, $\lambda=-\frac{i}{4}$. Further and similar calculations demonstrate that this is the only constraint [27].

Substitute this constraint into (68), we find that $\mu$ is a real number and for all $a, b \in \mathfrak{g}$,

$$
\jmath(\gamma(a) b)=-4 \mu \gamma(\jmath a)(\jmath b) .
$$

It concludes the proof of Proposition 6.

Let us analyze Proposition 6 further. If $\mu=0$, constraint (65) implies that $\gamma(a) b=0$ for all $a, b \in \mathfrak{g}$. Therefore, $\gamma=0$. However, the connection $\gamma$ is torsion-free. This implies that $[a, b]=0$. Therefore, the algebra $\mathfrak{h}=\mathfrak{g} \ltimes V$ is trivial. In particular, $\Lambda$ is central in the Gerstenhaber algebra $(\wedge \bullet \mathfrak{h}, \wedge, \llbracket-,-\rrbracket)$, and hence $(\Lambda, \phi=0)$ forms a compatible pair.

Therefore, whenever $\mathfrak{h}$ is non-abelian, we may assume that $\mu \neq 0$. In such case, if one multiplies the non-degenerate bilinear form $g$ on $\mathfrak{g}$ by the constant $-4 \mu$, then the inhomogeneity in equation (65) allows us to simplify this identity to

$$
\left(g^{-1} \omega\right)(\gamma(a) b)=\gamma\left(\left(g^{-1} \omega\right)(a)\right)\left(\left(g^{-1} \omega\right)(b)\right) .
$$

Now we could apply Proposition 6 and Theorem 2 to conclude the following.

THeOREM 5. Let $\mathfrak{g}$ be a Lie algebra with an invariant symplectic structure $\omega$ and non-degenerate bilinear form $g$. Let $V$ be its underlying vector space. Let $\gamma: \mathfrak{g} \rightarrow$ $\operatorname{End}(V)$ be a torsion-free flat connection and $\mathfrak{h}=\mathfrak{g} \ltimes_{\gamma} V$ the associated semi-direct product. Then $\mathfrak{h}$ has a natural complex structure $J$, a symplectic structure $\Omega$ and a pseudo-metric $\Delta$. If this triple forms a pseudo-Kähler structure and if

$$
\left(g^{-1} \omega\right)(\gamma(a) b)=\gamma\left(\left(g^{-1} \omega\right)(a)\right)\left(\left(g^{-1} \omega\right)(b)\right) .
$$

then there exists a deformation from the complex structure $J$ to a symplectic structure $\Omega_{2}$ such that $\mathrm{DGA}(J)$ is isomorphic to $\operatorname{DGA}\left(\Omega_{2}\right)$.

6. Low-dimension examples. According to Andranda [1], there are three nontrivial four-dimensional complex symplectic algebras. Let $e_{1}, e_{2}$ be a basis of $\mathfrak{g}$ and $v_{1}, v_{2}$ be a basis for $V$ such that

$$
J e_{1}=v_{1}, \quad J e_{2}=v_{2} .
$$

Let $e^{1}, e^{2}$ and $v^{1}, v^{2}$ be the dual bases. We choose the symplectic structure $\omega$ and the pseudo-metric $g$ on the algebra $\mathfrak{g}$ to be

$$
\omega=e^{1} \wedge e^{2}, \quad g=e^{1} \otimes e^{2}+e^{2} \otimes e^{1} .
$$


It follows that

$$
\jmath=g^{-1} \omega=e^{1} \otimes e_{1}-e^{2} \otimes e_{2} .
$$

The natural symplectic form and metric on $\mathfrak{g} \ltimes V$ are respectively

$$
\Omega=e^{1} \wedge e^{2}+v^{1} \wedge v^{2}, \quad \Delta=e^{1} \otimes e^{2}+e^{2} \otimes e^{1}+v^{1} \otimes v^{2}+v^{2} \otimes v^{1} .
$$

Moreover, let $z_{1}=\frac{1}{2}\left(e_{1}-i v_{1}\right)$ and $z_{2}=\frac{1}{2}\left(e_{2}-i v_{2}\right), z^{1}=e^{1}+i v^{1}$, and $z^{2}=e^{2}+i v^{2}$, then

$$
\begin{gathered}
\Omega_{1}=-e^{1} \wedge v^{2}-v^{1} \wedge e^{2}=\frac{1}{2 i}\left(z^{1} \wedge z^{2}-\bar{z}^{1} \wedge \bar{z}^{2}\right) \\
\Omega_{2}=e^{1} \wedge e^{2}-v^{1} \wedge v^{2}=\frac{1}{2}\left(z^{1} \wedge z^{2}+\bar{z}^{1} \wedge \bar{z}^{2}\right) \\
\Omega_{3}=e^{1} \wedge e^{2}+v^{1} \wedge v^{2}=\frac{1}{2}\left(z^{1} \wedge \bar{z}^{2}+\bar{z}^{1} \wedge z^{2}\right) \\
\Omega_{4}=e^{1} \wedge v^{2}-v^{1} \wedge e^{2}=\frac{i}{2}\left(z^{1} \wedge \bar{z}^{2}-\bar{z}^{1} \wedge z^{2}\right) .
\end{gathered}
$$

In particular,

$$
\begin{aligned}
\Omega_{c}=\Omega_{1}+i \Omega_{2}=i z^{1} \wedge z^{2}, & \Lambda=\Omega_{c}^{-1}=i z_{1} \wedge z_{2} \\
\Omega_{3}^{-1}=2\left(z_{2} \wedge \bar{z}_{1}+\bar{z}_{2} \wedge z_{1}\right), & \Omega_{4}^{-1}=2 i\left(z_{2} \wedge \bar{z}_{1}-\bar{z}_{2} \wedge z_{1}\right) .
\end{aligned}
$$

6.1. Example 1. When the two-dimensional Lie algebra $\mathfrak{g}$ is abelian, the only non-trivial object in constructing a four-dimensional semi-direct product in this case is the torsion-free flat connection $\gamma$. It is determined by the identities,

$$
\gamma\left(e_{1}\right) v_{1}=v_{2}, \quad \gamma\left(e_{1}\right) v_{2}=0, \quad \gamma\left(e_{2}\right)=0 .
$$

Equivalently, the only non-trivial structure equation for $\mathfrak{h}=\mathfrak{g} \ltimes V$ is

$$
\llbracket e_{1}, v_{1} \rrbracket=v_{2} .
$$

The dual structure equation is $d v^{2}=-e^{1} \wedge v^{1}$. Therefore, it is apparent that $\Omega_{4}$ is closed, and hence $\mathfrak{h}$ has a natural pseudo-Kähler metric.

As $\jmath e_{1}=e_{1}$ and $\jmath e_{2}=-e_{2}$, Proposition 6 is solved when $\mu=\frac{1}{4}$. By the expressions in (82),

$$
\phi=-\frac{i}{4} \Omega_{3}^{-1}+\frac{1}{4} \Omega_{4}^{-1}=\frac{1}{4}\left(\Omega_{4}^{-1}-i \Omega_{3}^{-1}\right)=i z_{1} \wedge \bar{z}_{2} .
$$

Therefore by Theorem 5, for the complex structure $J$ in (77) and the symplectic structure $\Omega_{2}$ in $(79), \operatorname{DGA}\left(\Omega_{2}\right)$ and $\operatorname{DGA}(J)$ are isomorphic and they exist in one generalized deformation class.

Indeed, for this particular example, the algebraic $\mathfrak{h}$ is the covering space of the Kodaira-Thurston surface. It is known that all the concerned cohomology spaces are given by invariant objects. Therefore, we may also apply Theorem 4 on manifold level, and recovers a key result obtained by ad hoc computation in [26]. 
6.2. Example 2. In this example, the algebra $\mathfrak{g}$ is solvable, with structure equation $\left[e_{1}, e_{2}\right]=e_{2}$. The connection $\gamma$ is given by

$$
\gamma\left(e_{1}\right) v_{1}=-v_{1}, \quad \gamma\left(e_{1}\right) v_{2}=v_{2}, \quad \gamma\left(e_{2}\right)=0
$$

The structure equations for the semi-direct product $\mathfrak{h}$ are equivalently given by

$$
d e^{2}=-e^{1} \wedge e^{2}, \quad d v^{1}=e^{1} \wedge v^{2}, \quad d v^{2}=-e^{1} \wedge v^{2} .
$$

It follows that $\Omega_{4}$ is closed. Further, $\mu=-\frac{1}{4}$ solves the constraint in Proposition 6 , and $\phi=-i z_{2} \wedge \bar{z}_{1}$. Therefore, by Theorem 5 the complex structure $J$ is deformed to $\Omega_{2}$ via a holomorphic Poisson structure, and $\operatorname{DGA}(J)$ is isomorphic to $\operatorname{DGA}\left(\Omega_{2}\right)$.

6.3. Example 3. In this example, the algebra $\mathfrak{g}$ is solvable: $\left[e_{1}, e_{2}\right]=e_{2}$. The connection $\gamma$ is given by

$$
\gamma\left(e_{1}\right) v_{1}=-\frac{1}{2} v_{1}, \quad \gamma\left(e_{1}\right) v_{2}=\frac{1}{2} v_{2}, \quad \gamma\left(e_{2}\right) v_{1}=-\frac{1}{2} v_{2}, \quad \gamma\left(e_{2}\right) v_{2}=0
$$

On the semi-direct product the non-trivial structure equations become

$$
\left[e_{1}, e_{2}\right]=e_{2}, \quad\left[e_{1}, v_{1}\right]=-\frac{1}{2} v_{1}, \quad\left[e_{1}, v_{2}\right]=\frac{1}{2} v_{2}, \quad\left[e_{2}, v_{1}\right]=-\frac{1}{2} v_{2} .
$$

The dual equations are

$$
d e^{2}=-e^{1} \wedge e^{2}, \quad d v^{1}=\frac{1}{2} e^{1} \wedge v^{1}, \quad d v^{2}=-\frac{1}{2} e^{1} \wedge v^{2}+\frac{1}{2} e^{2} \wedge v^{1} .
$$

It follows that $d \Omega_{4}=2 v^{1} \wedge e^{1} \wedge e^{2}$. In particular, Proposition 6 and Theorem 5 are not applicable. In terms of complex frames, we have

$$
\llbracket z_{1}, z_{2} \rrbracket=\frac{1}{2} z_{2}, \quad d z^{1}=-\frac{1}{4} z^{1} \wedge \bar{z}^{1}, \quad d z^{2}=-\frac{1}{4}\left(z^{1}+\bar{z}^{1}\right) \wedge z^{2}-\frac{1}{4} z^{1} \wedge\left(z^{2}+\bar{z}^{2}\right) .
$$

From the differentials, we further obtain that

$$
\llbracket z_{1}, \bar{z}^{1} \rrbracket=\frac{1}{4} \bar{z}^{1}, \quad \llbracket z_{1}, \bar{z}^{2} \rrbracket=-\frac{1}{4} \bar{z}^{2}, \quad \llbracket z_{2}, \bar{z}^{2} \rrbracket=\frac{1}{4} \bar{z}^{1} .
$$

Taking the complex conjugation, and then the dual expression is

$$
\bar{\partial} z_{1}=-\frac{1}{4} \bar{z}^{1} \wedge z_{1}-\frac{1}{4} \bar{z}^{2} \wedge z_{2}, \quad \bar{\partial} z_{2}=\frac{1}{4} \bar{z}^{1} \wedge z_{2} .
$$

As an intermediate step, we put together the structure equation of $\operatorname{DGA}(J)$ on this particular algebra:

$$
\begin{aligned}
& \llbracket z_{1}, z_{2} \rrbracket=\frac{1}{2} z_{2}, \quad \llbracket z_{1}, \bar{z}^{1} \rrbracket=\frac{1}{4} \bar{z}^{1}, \quad \llbracket z_{1}, \bar{z}^{2} \rrbracket=-\frac{1}{4} \bar{z}^{2}, \quad \llbracket z_{2}, \bar{z}^{2} \rrbracket=\frac{1}{4} \bar{z}^{1} \\
& \bar{\partial} z_{1}=-\frac{1}{4} \bar{z}^{1} \wedge z_{1}-\frac{1}{4} \bar{z}^{2} \wedge z_{2}, \quad \bar{\partial} z_{2}=\frac{1}{4} \bar{z}^{1} \wedge z_{2}, \quad \bar{\partial} \bar{z}^{2}=-\frac{1}{2} \bar{z}^{1} \wedge \bar{z}^{2}
\end{aligned}
$$

On the other hand,

$$
\Omega_{2}\left(e_{1}\right)=e^{2}, \quad \Omega_{2}\left(e_{2}\right)=-e^{1}, \quad \Omega_{2}\left(v_{1}\right)=-v^{2}, \quad \Omega_{2}\left(v_{2}\right)=v^{1} .
$$


Then the linear isomorphism $\Omega_{2}$ take the Lie bracket on vectors in (83) to a Lie bracket on forms.

$$
\llbracket e^{1}, e^{2} \rrbracket=-e^{1}, \quad \llbracket e^{2}, v^{2} \rrbracket=\frac{1}{2} v^{2}, \quad \llbracket e^{2}, v^{1} \rrbracket=\frac{1}{2} v^{1}, \quad \llbracket e^{1}, v^{2} \rrbracket=-\frac{1}{2} v^{1} .
$$

With respect to these Lie algebra structures, the first derived subalgebra $\mathfrak{h}^{1,0} \oplus \mathfrak{h}^{*(0,1)}$ is the three-dimensional Heisenberg algebra spanned by $e^{1}, v^{1}, v^{2}$ with $v^{1}$ being its center. In view of the exterior differential as given in (84), $v^{1}$ is not closed in the differential Gerstenhaber algebra of the symplectic structure $\Omega_{2}$.

On the other hand, from (86), we find that the first derived subalgebra in $\operatorname{DGA}(J)$ is the three-dimensional Heisenberg algebra spanned by $z_{2}, \bar{z}^{1}, \bar{z}^{2}$ with $\bar{z}^{1}$ being its center. In view of $(87), \bar{z}^{1}$ is $\bar{\partial}$-closed.

Since the center of the derived subalgebra of $\operatorname{DGA}(J)$ is $\bar{\partial}$-closed and that of $\operatorname{DGA}\left(\Omega_{2}\right)$ is not $d$-closed, these two DGAs could not be quasi-isomorphic

REMARK. Given the definition of $\Omega_{1}$ in (78), it is apparent that $\mathfrak{g}$ and $V$ are Lagrangian with respect to $\Omega_{1}$. As $J \mathfrak{g}=V$ and $J V=\mathfrak{g}$, the complex structure $J$ and the complex symplectic structure is special Lagrangian in the sense of [7, Definition].

Let $\gamma^{*}$ be the dual representation of $\gamma$, then one obtains the dual semi-direct product $\widehat{\mathfrak{h}}=\mathfrak{g} \ltimes_{\gamma^{*}} V^{*}$. Through this space as an intermediate object, it is provided in $\left[7\right.$, Theorem 5.2] that there is a natural isomorphism from $\operatorname{DGA}(J)$ to $\operatorname{DGA}\left(\Omega_{1}\right)$. See also [4]. Therefore, we have

$$
\operatorname{DGA}\left(\Omega_{1}\right) \cong \operatorname{DGA}(J) \cong \operatorname{DGA}\left(\Omega_{2}\right)
$$

\section{REFERENCES}

[1] A. Andrada, Hypersymplectic Lie algebras, J. Geom. Phys., 56 (2006), pp. 2039-2067.

[2] S. Barannikov and M. Kontsevich, Frobenius manifolds and formality of Lie algebras of polyvector fields, Internat. Math. Res. Notices, 4 (1998), pp. 201-215.

[3] C. Bartocci and E. Marci, Classification of Poisson surfaces, Commun. Contemp. Math., 7 (2005), pp. 89-95.

[4] O. Ben-BAssat, Mirror symmetry and generalized complex manifolds. I 6 II. The transform on vector bundles, spinors, and branes, J. Geom. Phys., 56 (2006), pp. 533-558, 1096-1115.

[5] H.-D. CAO AND J. Zhou, On quasi-isomorphic DGBV algebras, Math. Ann., 326 (2003), pp. $459-478$.

[6] R. Cleyton, J. Lauret, and Y. S. Poon, Weak mirror symmetry of Lie algebras, J. Symplectic Geom., 8 (2010), pp. 37-55.

[7] R. Cleyton, G. Ovando, and Y. S. Poon, Weak mirror symmetry of complex symplectic algebras, J. Geom. Phys., 61 (2011), pp. 1553-1563.

[8] S. Console And A. Fino, Dolbeault cohomology of compact nilmanifolds, Transform. Groups., 6 (2001), pp. 111-124.

[9] S. Console, A. Fino, And Y. S. Poon, Stability of abelian complex structures, International J. Math., 17 (2006), pp. 401-416.

[10] L. A. Cordero, M. Fernández, A. Gray and L. Ugarte, Compact nilmanifolds with nilpotent complex structures: Dolbeault cohomology, Trans. Amer. Math. Soc., 352 (2000), pp. 5405-5433.

[11] T. Courant, Dirac structures, Trans. Amer. Math. Soc., 319 (1990), pp. 631-661.

[12] K. Dabrowski, Moduli spaces for Hopf surfaces, Math. Ann., 259 (1982), pp. 201-225.

[13] D. Fiorenza And M. Manetti, Formality of Koszul brackets and deformations of holomorphic Poisson manifolds, preprint, arXiv:1109.4309v2.

[14] P. Gauduchon, Hermitian connections and Dirac operators, Bollettino U.M.I., 11B (1997), pp. $257-288$.

[15] R. Goto, Deformations of generalized complex and generalized Kähler structures, J. Differential Geom., 84 (2010), pp. 525-560.

[16] G. Grantcharov, C. McLaughlin, H. Pedersen, and Y. S. Poon, Deformations of Kodaira manifolds, Glasgow Math. J., 46 (2004), pp. 259-281. 
[17] M. Gualtieri, Generalized complex geometry, Ann. of Math., 174 (2011), pp. 75-123.

[18] N. J. Hitchin, Generalized Calabi-Yau manifolds, Quart. J. Math., 54 (2003), pp. 281-308.

[19] N. J. Hitchin, Bihermitian metrics on Del Pezzo surfaces, J. Symplectic Geom., 5 (2007), pp. $1-7$.

[20] N. J. Hitchin, Deformation of holomorphic Poisson manifolds, Mosc. Math. J., 12 (2012), pp. 567-591.

[21] Z. J. Liu, A. Weinstein, And P. Xu, Manin triples for Lie bialgebroids, J. Differential Geom., (1997), pp. 547-574.

[22] K. MacKenzie, General Theory of Lie Groupoids and Lie Algebroids, London Math. Soc., Lecture Notes Series, 213 (2005), Cambridge University Press.

[23] S. A. Merkulov, Frobenius $\infty$ invariants of homotopy Gerstenhaber algebras, I, Duke Math. J., 105 (2000), pp. 411-461.

[24] S. A. Merkulov, A note on extended complex manifolds. Symplectic and contact topology: interactions and perspectives (Toronto, ON/Montreal, QC, 2001), pp. 145-155, Fields Inst. Commun., 35, Amer. Math. Soc., Providence, RI, 2003.

[25] K. Nomizu, On the cohomology of compact homogenous spaces of nilpotent Lie groups, Ann. Math., 59 (1954), pp. 531-538.

[26] Y. S. Poon, Extended deformation of Kodaira surfaces, J. reine angew. Math., 590 (2006), pp. $45-65$.

[27] B. Rolle, Construction of weak mirror pairs by deformations, PhD. Thesis, Univeristy of California at Riverside (2011).

[28] S. Rollenske, Lie algebra Dolbeault cohomology and small deformations of nilmanifolds, J. London. Math. Soc. (2), 79 (2009), pp. 346-362. 